\title{
Properties and geoeffectiveness of magnetic clouds in the rising, maximum and early declining phases of solar cycle 23
}

\author{
K. E. J. Huttunen ${ }^{1}$, R. Schwenn ${ }^{2}$, V. Bothmer ${ }^{2}$, and H. E. J. Koskinen ${ }^{1,3}$ \\ ${ }^{1}$ Department of Physical Sciences, Theoretical Physics Division, P.O. Box 64, FIN-00014 University of Helsinki, Finland \\ ${ }^{2}$ Max-Planck-Institut für Sonnensystemforschung, D-37191, Germany \\ ${ }^{3}$ Finnish Meteorological Institute, P.O. Box 503, 00101 Helsinki, Finland
}

Received: 30 March 2004 - Revised: 1 October 2004 - Accepted: 12 October 2004 - Published: 28 February 2005

\begin{abstract}
The magnetic structure and geomagnetic response of 73 magnetic clouds (MC) observed by the WIND and ACE satellites in solar cycle 23 are examined. The results have been compared with the surveys from the previous solar cycles. The preselected candidate MC events were investigated using the minimum variance analysis to determine if they have a flux-rope structure and to obtain the estimation for the axial orientation $\left(\theta_{C}, \phi_{C}\right)$. Depending on the calculated inclination relative to the ecliptic we divided MCs into "bipolar" $\left(\theta_{C}<45^{\circ}\right)$ and "unipolar" $\left(\theta_{C}>45^{\circ}\right)$. The number of observed MCs was largest in the early rising phase, although the halo CME rate was still low. It is likely that near solar maximum we did not identify all MCs at $1 \mathrm{AU}$, as they were crossed far from the axis or they had interacted strongly with the ambient solar wind or with other CMEs. The occurrence rate of MCs at $1 \mathrm{AU}$ is also modified by the migration of the filament sites on the Sun towards the poles near solar maximum and by the deflection of CMEs towards the equator due to the fast solar wind flow from large polar coronal holes near solar minimum. In the rising phase nearly all bipolar MCs were associated with the rotation of the magnetic field from the south at the leading edge to the north at the trailing edge. The results for solar cycles 21-22 showed that the direction of the magnetic field in the leading portion of the MC starts to reverse at solar maximum. At solar maximum and in the declining phase (2000-2003) we observed several MCs with the rotation from the north to the south. We observed unipolar (i.e. highly inclined) MCs frequently during the whole investigated period. For solar cycles 21-22 the majority of MCs identified in the rising phase were bipolar while in the declining phase most MCs were unipolar. The geomagnetic response of a given MC depends greatly on its magnetic structure and the orientation of the sheath fields. For each event we distinguished the effect of the sheath fields and the MC fields. All unipolar MCs with magnetic field southward at the axis were geoeffective $\left(D_{s t}<-50 \mathrm{nT}\right)$ while those with
\end{abstract}

Correspondence to: K. E. J. Huttunen

(emilia.huttunen@helsinki.fi) the field pointing northward did not cause magnetic storms at all. About half of the all identified MCs were not geoffective or the sheath fields preceding the MC caused the storm. MCs caused more intense magnetic storms $\left(D_{s t}<-100 \mathrm{nT}\right)$ than moderate magnetic storms $\left(-50 \mathrm{nT} \geq D_{s t} \geq-100 \mathrm{nT}\right)$.

Key words. Interplanetary physics (Interplanetary magnetic fields) - Magnetospheric physics (Solar windmagnetosphere interactions) - Solar physics, astrophysics and astronomy (Flares and mass ejections)

\section{Introduction}

Manifestations of coronal mass ejections (CMEs) are frequently observed in the solar wind near $1 \mathrm{AU}$ and are commonly called interplanetary coronal mass ejections (ICMEs). The term magnetic cloud (MC) is used to characterize an ICME having a specific configuration in which the magnetic field strength is higher than the average, the magnetic field direction rotates smoothly through a large angle, and the proton temperature is low, Burlaga et al. (1981); Klein and Burlaga (1982); Gosling (1990). Because of the high magnetic field strength and low proton temperatures MCs have values of plasma beta significantly lower than 1 . Near 1 AU MCs have enormous radial sizes $(0.28 \mathrm{AU})$, with an average duration of $27 \mathrm{~h}$, an average peak magnetic field strength of $\sim 18 \mathrm{nT}$ and the average solar wind speed $420 \mathrm{~km} / \mathrm{s}$, Klein and Burlaga (1982); Lepping and Berdichevsky (2000). The expansion of a MC produces strongly decreasing density and temperature with the radial distance from the Sun and declining profiles of speed, magnetic field and pressure, Burlaga and Behannon (1982); Gosling (1990); Bothmer and Schwenn (1998). The interaction with the ambient solar wind may prevent the expansion that leads to a smaller diameter and larger densities and temperatures at $1 \mathrm{AU}$ than in an average MC. Goldstein (1983) first suggested that MCs are force-free magnetic field configurations $(\nabla \times \boldsymbol{B}=\alpha(r) \boldsymbol{B})$. 


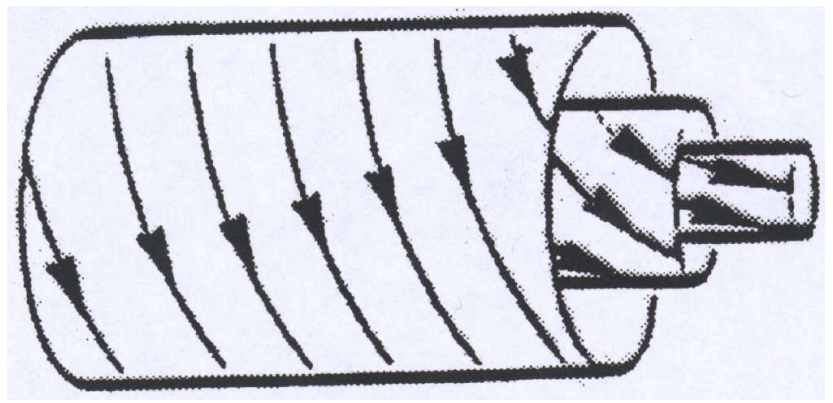

Fig. 1. The flux rope of type SWN showing the rotation of the magnetic field vector from the south to the west at the MC-axis and finally to the north at the trailing edge of the MC (Bothmer and Rust, 1997).

A few years later Burlaga (1988) showed that a constant $\alpha$ describes satisfactorily the magnetic field changes when a MC moves past a spacecraft. The constant $\alpha$ solution for a cylindrical symmetric force-free equation was given by Lundquist (1950):

$B_{R}=0, B_{A}=B_{0} J_{0}(\alpha r), B_{T}=H B_{0} J_{1}(\alpha r)$,

where $B_{R}, B_{A}$ and $B_{T}$ are the radial, axial and tangential components of the magnetic field. $B_{0}$ is the maximum of the magnetic field strength, $r$ is the radial distance from the axis, $\alpha$ is a constant related to the size of a flux rope, $J_{0}$ and $J_{1}$ are Bessel functions and $H= \pm 1$ defines the sign of the magnetic helicity Elsässer (1958); Berger and Field (1984).

The four possible flux-rope configurations, as predicted from Eq. (1), have been confirmed to occur in the solar wind, Bothmer and Schwenn (1994); Bothmer and Schwenn (1998). The axis of an MC $\left(\phi_{C}, \theta_{C}\right)$ can have any orientation with respect to the ecliptic plane and depending on the observed directions of the magnetic field at the front boundary, at the axis and at the end boundary eight flux rope categories are often used to classify MCs, Bothmer and Schwenn (1994); Bothmer and Schwenn (1998); Mulligan et al. (1998):

- Bipolar MCs (low inclination), $\theta_{C}<45^{\circ}$ : Following the terminology by Mulligan et al. (1998) the MCs with the axis lying near the ecliptic plane are called bipolar, as the $Z$ component of the terrestrial magnetic field changes sign during the passage of an MC. Figure 1, adopted from Bothmer and Rust (1997), shows a sketch of the flux rope category called SWN. In the SWN-type $\mathrm{MC}$ the magnetic field vector rotates from the south (S) at the leading edge to the north $(\mathrm{N})$ at the trailing edge, being westward (W) at the axis. Similarly, the three other categories are SEN (E=east), NES and NWS.

- Unipolar MCs (high inclination), $\theta_{C}>45^{\circ}$ : The MCs that have the axis highly inclined to the ecliptic are called unipolar, as the $Z$-component has the same sign during the MC. The magnetic field is observed to rotate from the west (east) at the leading edge to the east (west) at the trailing edge, pointing either south or north at the axis. These changes correspond to the flux-rope types: WNE, ESW, ENW and WSE.

When viewed by an observer looking towards the Sun (positive axis direction) the counterclockwise magnetic field rotation is defined as right-handed (SWN, NES, ENW and WSE types) and the clockwise rotation as left-handed (NWS, SEN, WNE, and ESW types). The handedness can be determined from the parameters $H$ and $\phi_{c}$ with the formula, $C=\operatorname{sgn}\left(\sin \phi_{c}\right) \times H$, such that $C=-1$ is for a left-handed MC and $C=+1$ is for a right-handed MC (Lynch et al., 2003). The studies of MCs during different activity phases for solar cycles 21-22 revealed systematic variations in the preferred flux rope types, Bothmer and Rust (1997); Bothmer and Schwenn (1998); Mulligan et al. (1998): In the rising phase of odd (even) solar cycles the magnetic field in MCs rotates predominantly from the south to the north (from the north to the south) and during the years of high solar activity both SN and NS type MCs are observed. Additionally, Mulligan et al. (1998) found for the years 1979-1988 that unipolar MCs were most frequently observed in the declining phase of the solar activity cycle. At solar minimum and in the rising phase most MCs were bipolar.

MCs have been studied intensively since their discovery, as they are important drivers of magnetic storms, e.g. Tsurutani et al. (1988); Zhang et al. (1988); Gosling et al. (1991). A magnetic storm is defined as a world wide depression in the horizantal component of the magnetic field that is caused by the enhanced ring current (Gonzalez et al., 1994). The variations in the ring current are recorded by the 1-h $D_{s t}$ index, e.g. Mayaud (1980). The key parameters that control the solar wind magnetospheric coupling are the strength and the direction of the interplanetary magnetic field (IMF). For example, intense magnetic storms $\left(D_{s t}<-100 \mathrm{nT}\right)$ are caused by an IMF southward component stronger than $10 \mathrm{nT}$ at least for $3 \mathrm{~h}$ (Gonzalez and Tsurutani, 1987). Solar wind speed and density also play a role in a formation of the ring current, though their exact role is still controversial, Gonzalez and Tsurutani (1987); Fenrich and Luhmann (1998); Wang et al. (2003a). The geomagnetic response of a certain MC depends greatly on its flux-rope structure, e.g. Zhang et al. (1988); Bothmer (2003). In some cases MCs cause major magnetic storms, for example, Bastille day storm on 15-16 June 2000 (Lepping et al., 2001) while in other cases the magnetic field remains mainly northward during the $\mathrm{MC}$ and no geomagnetic activity follows. A magnetic storm can also be caused by the sheath of heated and compressed solar wind plasma piled up in front of the CME ejecta (Tsurutani et al., 1988).

In this study we have performed the first extensive survey of the magnetic structure and the geomagnetic response of MCs identified during solar cycle 23 . The investigated period covers the rising phase of solar activity (1997-1999), solar maximum (2000) and the early declining phase (20012003) when defined by the yearly sunspot number. The purpose of this study is to examine whether the variations of the 
magnetic structure of MCs with solar activity found for the previous solar cycles (21-22) hold true also for solar cycle 23. During the investigated period we have continuous solar wind measurements at $1 \mathrm{AU}$ from WIND and ACE spacecraft, providing a larger set of MCs than was available for the previous solar cycles. We also present a detailed analysis of the geomagnetic response of the MCs, distinguishing the effect of sheath fields and MC fields as a storm drivers. The properties of MCs during solar cycle 23 have been surveyed by Lynch et al. (2003) and Wu et al. (2003). The Lynch et al. (2003) study covers only a three and one-half year period and concentrates on the plasma composition of MCs. The Wu et al. (2003) paper shortly summarizes the occurrence rate and geoeffects of MCs reported in the WIND list at http://lepmfi.gsfc.nasa.gov/mfi/mag_cloud_pub1.html. In Sect. 2 we present the method to identify MCs from the solar wind data and how the axial orientation was estimated. In Sect. 3 we show statistical results and in Sect. 4 we discuss the geoeffectiveness of MCs. In Sects. 5 and 6 we discuss and summarize the results.

\section{Identification of MCs and determination of their flux- rope type}

We have identified MCs using magnetic field and plasma measurements from WIND (January 1997-February 1998) and ACE (March 1998-December 2003). We first performed a visual inspection of the data to find the candidate MCs. The intervals of bidrectional streaming of solar wind suprathermal electrons (BDE) along magnetic field lines is often used to identify MCs, as this feature is considered to represent a closed magnetic field configuration (Bame et al., 1981; Gosling, 1990). However, as the interpretation of the BDE intervals is not unambiguous and BDE are present also in ICMEs without the MC structure, we did not use them as a $\mathrm{MC}$ signature. In this study the criteria to define an $\mathrm{MC}$ is based on the smoothness of the rotation in the magnetic field direction confined to one plane (see below). Additionally, we required that an $\mathrm{MC}$ must have the average values of plasma beta less than 0.5 , the maximum value of the magnetic field at least $8 \mathrm{nT}$ and the duration at least $6 \mathrm{~h}$. With the last two criteria we wanted to remove the ambiguity in identifying small and weak MCs. As a consequence, we are likely to miss MCs that have been crossed far from the axis. There is often a disagreement in the number of MCs identified in different studies because there is no unique and fully objective way to identify an MC in the solar wind (discussion, for example, in a poster by Shinde et al. at the fall AGU meeting, 2003).

All selected events were investigated by analyzing 1-h magnetic field data with the minimum variance analysis (MVA) (Sonnerup and Cahill, 1967), where MCs are identified from the smooth rotation of the magnetic field vector in the plane of the maximum variance (Klein and Burlaga, 1982). For MCs with durations of $12 \mathrm{~h}$ or less we performed MVA using 5-min (WIND) or 4-min (ACE) averaged data.
The detailed description of the method is found in the appendix of Bothmer and Schwenn (1998). The MVA method can be applied satisfyingly to the directional changes of the magnetic field vector exceeding $\sim 30^{\circ}$. The large ratio of the intermediate eigenvalue $\lambda_{2}$ to the minimum eigenvalue $\lambda_{3}$ indicates that the eigenvectors are well defined. We required that $\lambda_{2} / \lambda_{3}$ is greater than 2 , based on the analysis of Lepping and Behannon (1980). $B_{X}^{*}, B_{Y}^{*}$, and $B_{Z}^{*}$ correspond to the magnetic field components in the directions of maximum, intermediate and minimum variance. The MVA analysis provides us with the estimation of the orientation of the MC axis $\left(\phi_{C}, \theta_{C}\right) . \theta$ and $\phi$ are the latitudinal and longitudinal angels of the magnetic field vector in solar ecliptic coordinates; $\theta=90^{\circ}$ is defined northward and $\phi=90^{\circ}$ is defined eastward. The MC axis orientation corresponds to the direction of the intermediate variance that is seen from Eq. (1) as the axial component is zero at the boundaries of the MC. The radial component corresponds to the minimum variance direction and the azimuthal component corresponds to the maximum variance direction. The boundaries of MCs were determined by solar wind signatures (start of the smooth rotation of the magnetic field vector, drop in plasma beta, and plasma and field discontinuities) and by the eigenvalue ratio. In those cases where the boundaries defined by the different signatures disagreed we used the magnetic field rotation.

There are various other methods to model MCs. Lepping et al. (1990) have developed an algorithm to fit the magnetic field data to the Lundquist solution that reproduces well the observed directional changes of the magnetic field but often the magnetic field strength profile is not so well fitted. To improve the results the kinematic effects, such as the expansion and the assumptions of non-symmetric and non-force free topologies are used in some models, e.g. Farrugia et al. (1993); Marubashi (1997); Osherovich and Burlaga (1997); Mulligan and Russell (2001); Hidalgo et al. (2002a); Hidalgo et al. (2002b).

Figure 2 shows 1-h solar wind data and the calculated plasma beta during two MCs, one having the axis perpendicular to the ecliptic plane (left) and the other lying near the ecliptic plane (right). The bottom part of Fig. 2 shows the rotation of the magnetic field vector in the plane of maximum variance and in the plane of minimum variance. Both MCs are easily identified by the smooth rotation of the magnetic field direction, enhanced magnetic field magnitude and low plasma beta. The unipolar MC was observed by ACE on 19-21 March 2001. As seen from the Fig. 2 this MC has a flux-rope type WSE and the observed angular variation of the magnetic field is left-handed. The MVA method gives the eigenvalue ratio $\lambda_{2} / \lambda_{3}=52$, the angle between the first and the last magnetic field vectors $\chi=157^{\circ}$, and the orientation of the axis $\left(\phi_{C}, \theta_{C}\right)=\left(133^{\circ},-57^{\circ}\right)$. The $B_{z}$ component was southward almost during the whole passage of the MC (it caused a magnetic storm with the $D_{s t}$ minimum $-165 \mathrm{nT}$ ). The bipolar MC in Fig. 2 was observed by ACE on 20-21 August 1998. It belongs to flux rope category SWN and is right-handed. The MVA method gives the eigenvalue ratio $30, \chi=177^{\circ}$, and the orientation of the axis $\left(\phi_{C}, \theta_{C}\right)=\left(113^{\circ}\right.$, 

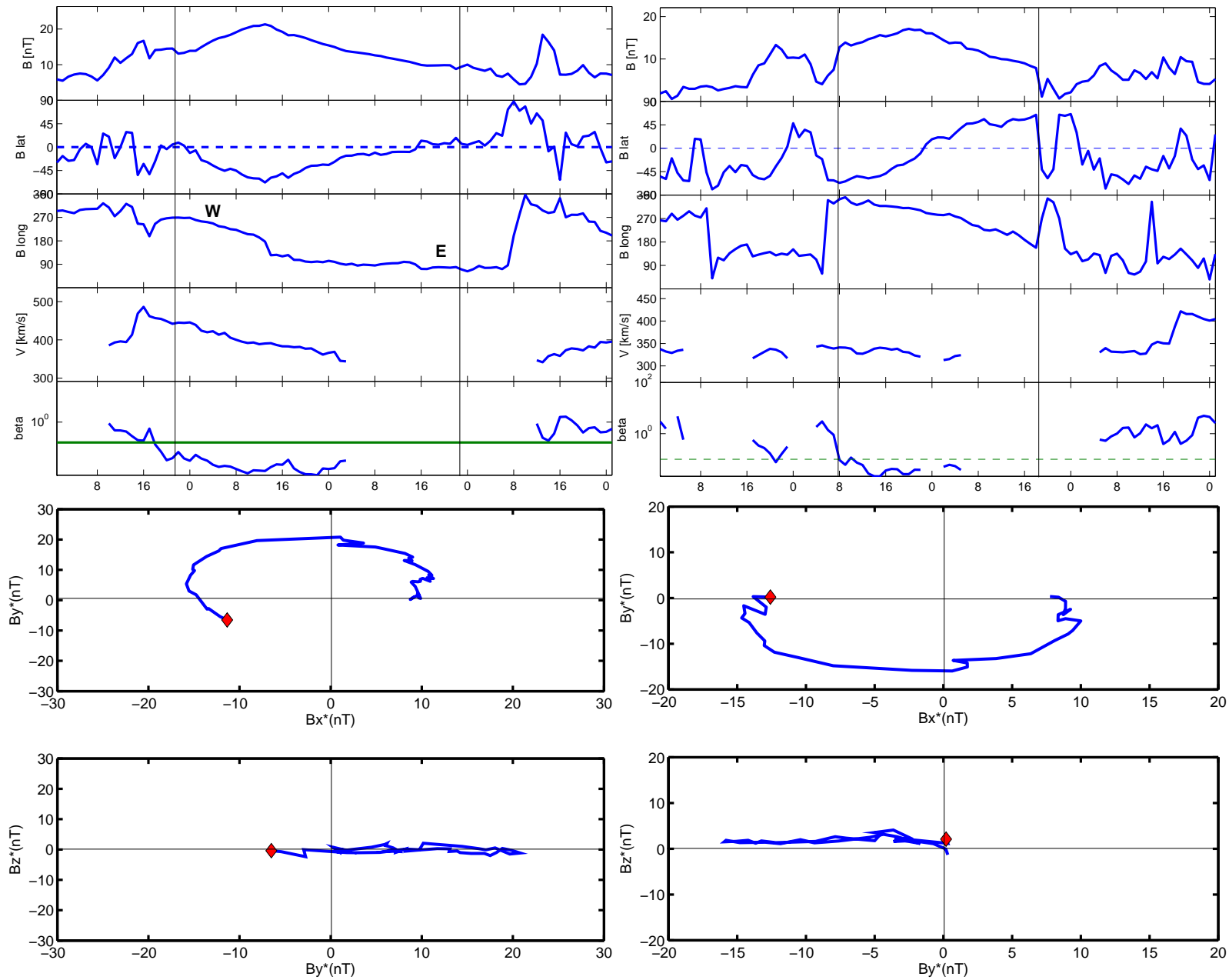

Fig. 2. Top part: Solar wind parameters during two MC events. Top to bottom: magnetic field strength, polar (Blat) and azimuthal (Blong) angles of the magnetic field vector in GSE coordinate system, solar wind speed and plasma beta. Left: 19-22 March 2001. Right: 19-22 August 1998. Two solid lines indicate the interval of an MC. Bottom part: the rotation of the magnetic field vector in the plane of maximum variance and in the plane of minimum variance. The diamond indicates the start of the rotation.

$-16^{\circ}$ ). For both MCs the hodograms show that in the plane of maximum variance the magnetic field rotates smoothly through a large angle and in the plane of minimum variance the magnetic field decreases/increases from about zero to the minimum/maximum value of the $B_{Y}^{*}$-component and then goes back to zero.

\section{Statistical results on MCs}

We have compared our statistical results to the results obtained in several other studies during solar cycle 23 and the previous solar cycles. The article, the period of the investigation, duration of the study in years (T), spacecraft used (S/C), and the total number of identified MCs are summarized in the Table 1. Bothmer and Rust (1997) and Bothmer and Schwenn (1998) identified MCs based on the minimum variance analysis, Mulligan et al. (1998) identified and classified MCs using the visual inspection of the data while Lynch et al. (2003) and Wu et al. (2003)/WIND list used the leastsquare fitting routine by Lepping et al. (1990).

\subsection{Magnetic cloud list}

Table 2 presents the $73 \mathrm{MCs}$ that we have identified from ACE and WIND solar wind data during the seven-year period (1997-2003). We have also included seven "cloud candidate" events for which the fitting with MVA was not successful (e.g. the eigenvalue ratio $<2$ or the directional change less than $30^{\circ}$ ) or that had large values of beta throughout the event. For example, 24-25 November 2001 and 23-24 May 2003 events exhibited very low plasma beta, but the organized rotation of the magnetic field was not observed. For the first event the complex magnetic structure probably results from the interaction of multiple fast halo CMEs that were detected by LASCO within a short time interval, Huttunen et al. (2002b); Wang et al. (2003b). 
Table 1. Summary of the five previous studies we have compared our statistical results. In Bothmer and Rust (1997) no duty cycle considerations are made. In Bothmer and Schwenn (1998) MCs were observed between 0.3-1 AU. The Wu et al. (2003) study covered the years 1996-2001. For 1995 and 2002 see the WIND magnetic cloud list.

\begin{tabular}{lllll}
\hline study & period & T & S/C & MC \\
\hline Bothmer and Rust (1997) & 1965-1993 & 28 & OMNI-data base & 67 \\
Bothmer and Schwenn (1998) & December 1974-July 1981 & 6.7 & Helios 1/2 & 45 \\
Mulligan et al. (1998) & 1979-1988 & 10 & Pioneer Venus Orbiter & 61 \\
Lynch et al. (2003) & February 1998-July 2001 & 3.5 & ACE & 56 \\
Wu et al. (2003)/WIND list & 1995-2002 & 8 & WIND & 71
\end{tabular}
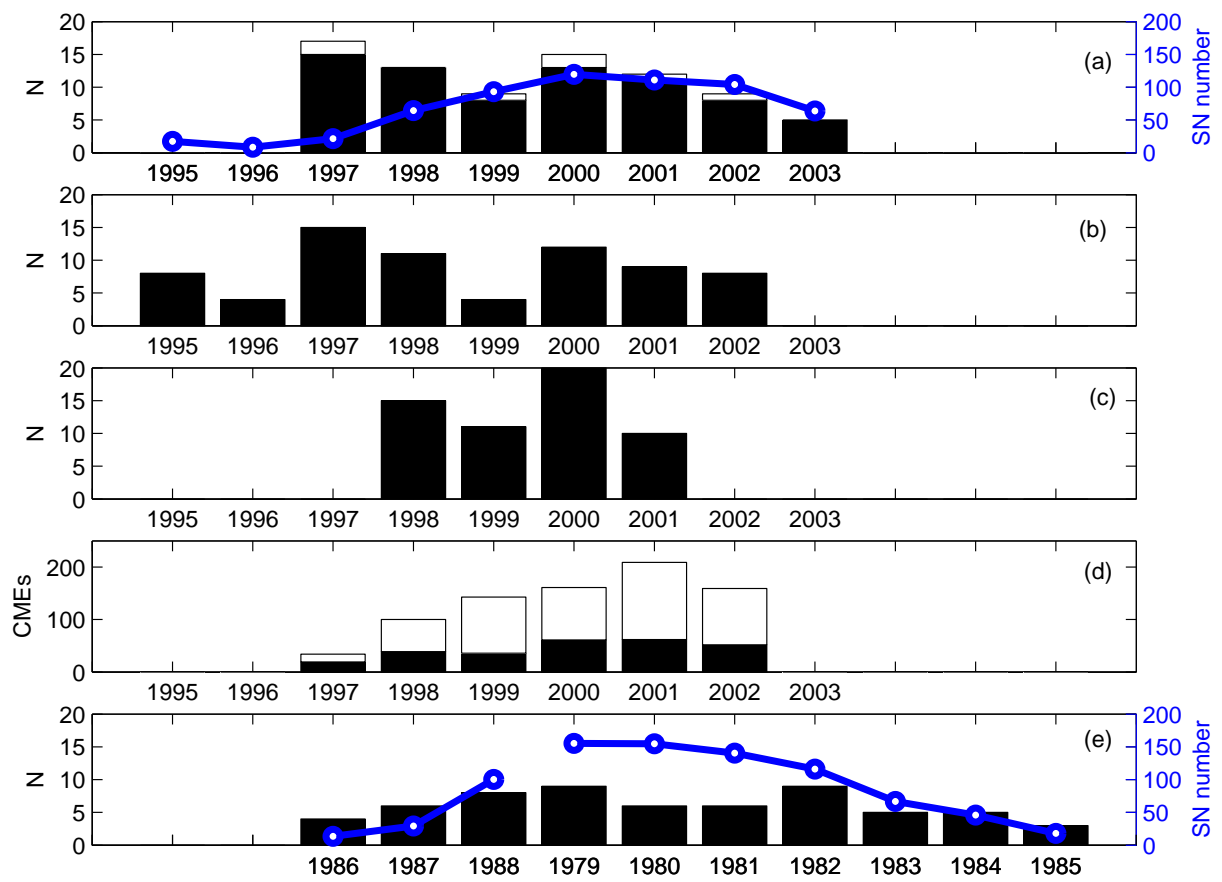

Fig. 3. Yearly number of observed MCs in our study (a), in Wu et al. (2003)/WIND list (b), and in Lynch et al. (2003) (c), the yearly number of departed full halo CMEs (black) and partial halo CMEs (white) (d), and yearly number of MCs given in Mulligan et al. (1998) (e). Note that in Lynch et al. (2003) the year 2001 presents only 7 months data of (January-July). The circles show the yearly sunspot number. The white portion in bars in (a) show the number of cloud candidate events. In (e) the years have been arranged to coincide with the years of approximately the same solar cycle phase in (a)-(d).

\subsection{Yearly magnetic cloud rate}

The histograms in Fig. 3 display the yearly number of MCs identified in our study (Fig. 3a), in Wu et al. (2003)/WIND list (Fig. 3b), and given in Lynch et al. (2003) (Fig. 3c). The circles show the yearly sunspot number and in Fig. 3a the white portions in bars show the "cloud candidate" events. The fourth Fig. 3d shows the yearly number of full (angular width $=360^{\circ}$ ) and partial (angular width $>120^{\circ}$ ) halo CMEs as reported in the LASCO coronal mass ejection catalogue (http://cdaw.gsfc.nasa.gov/CME_list). We have not made analysis as to whether these CMEs were front- or backside, but numbers shown give a rough estimate of the yearly changes in the number of CMEs that can encounter the Earth. Figure $3 \mathrm{e}$ shows the yearly number of MCs in Mulligan et al.
(1998). Note that in Fig. 3e we have arranged the time axis so that the years corresponding to about the same solar cycle phase coincide between Mulligan et al. (1998) and other studies.

Figure 3 a shows that we identified the largest number of MCs (15) just after solar minimum in 1997. The number of MCs was also high (13) in 1998 but there was a reduction to eight MCs in 1999. During solar maximum period (2000-2001) the MC rate was high, after which the number of identified MCs decreased. The yearly numbers given by Wu et al. (2003) show a similar trend. In 1999 they identified only four MCs. 


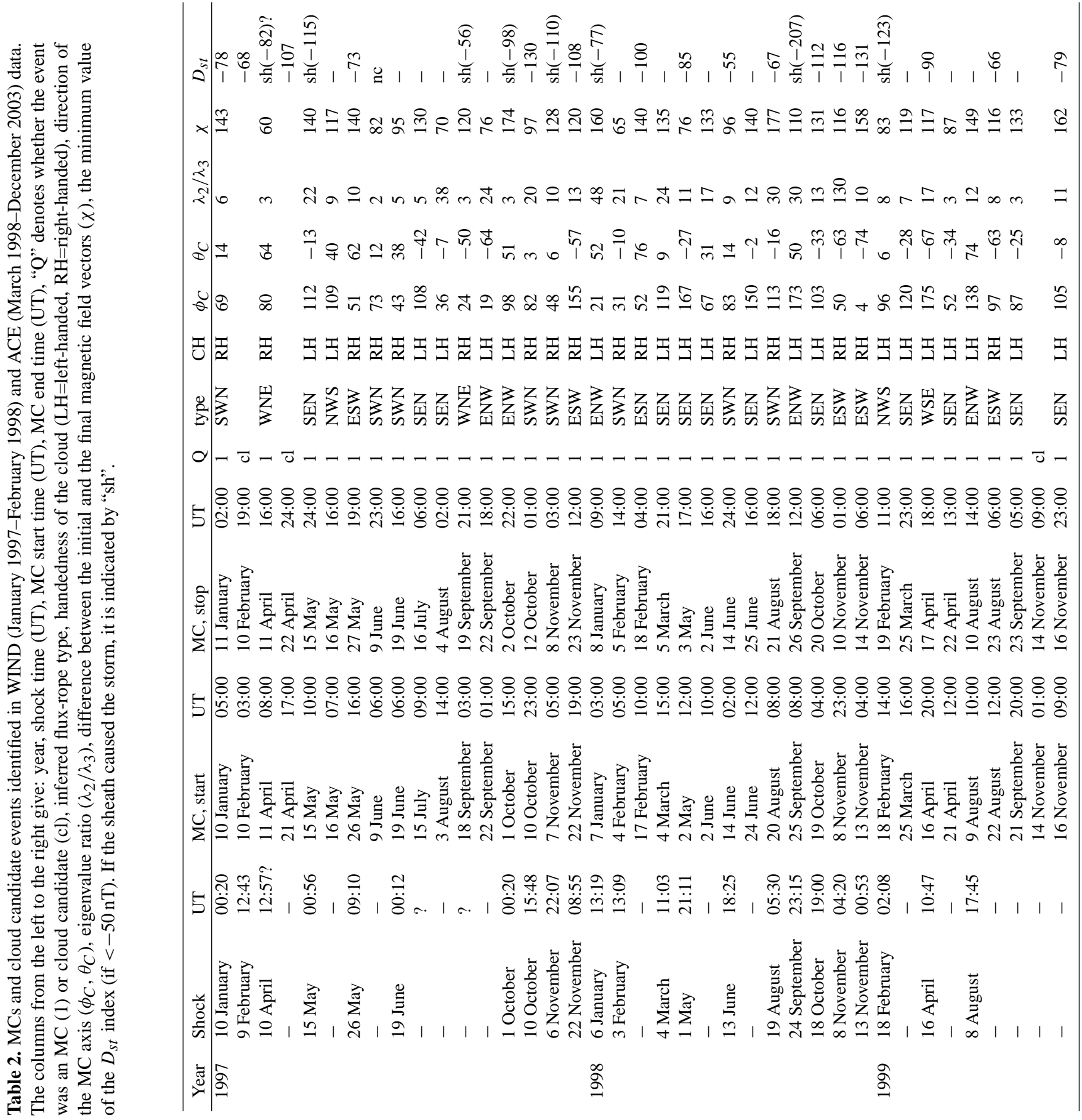




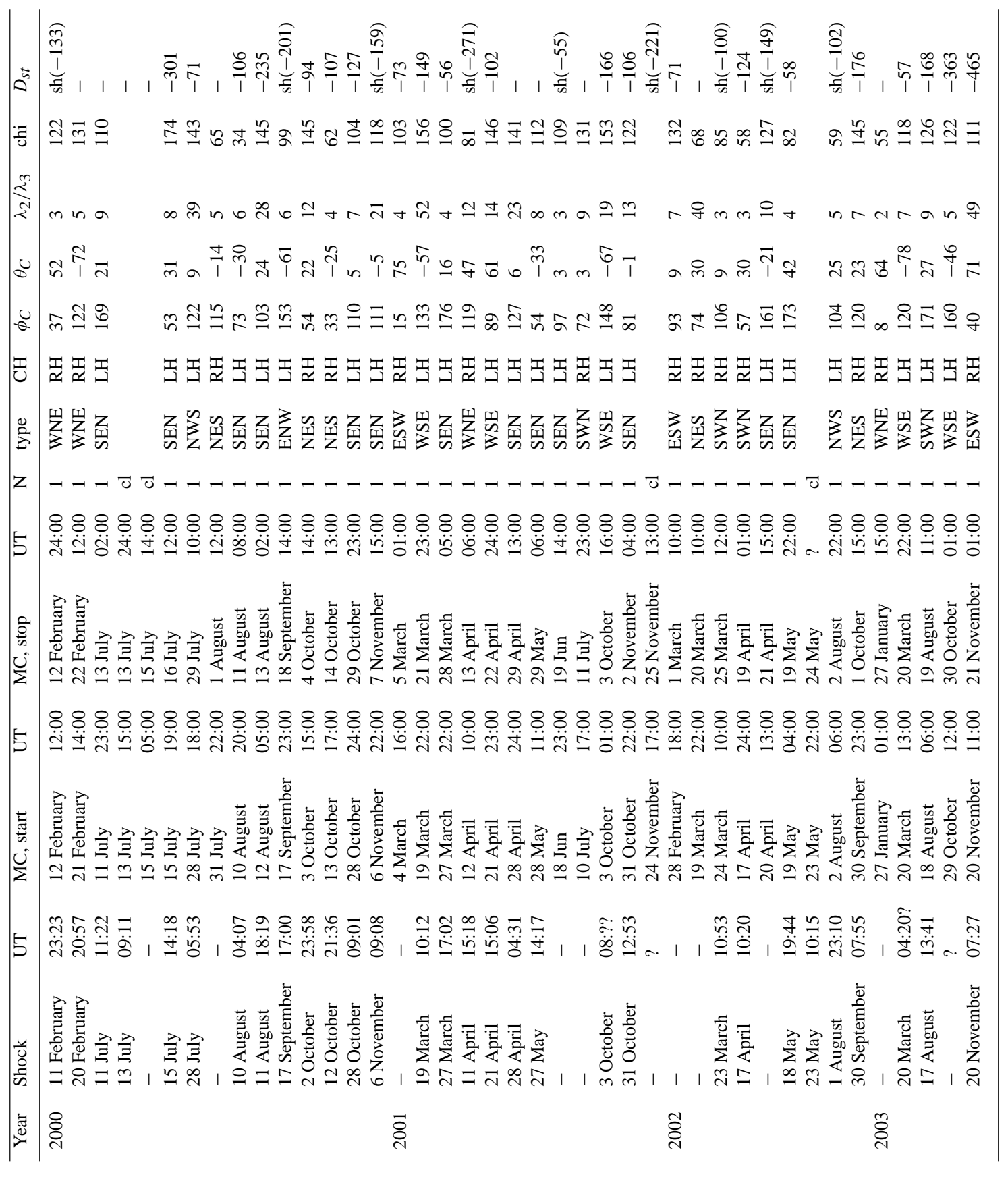




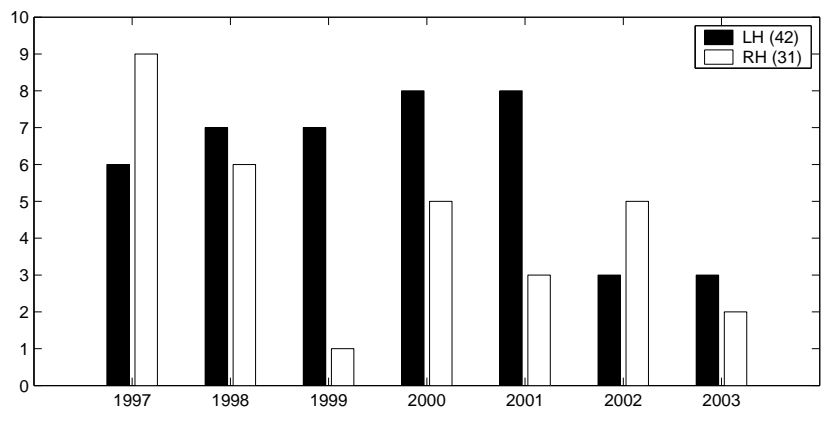

Fig. 4. Yearly distribution of left-handed (black) and right-handed MCs (white).

Three of the MCs that are included in our list in 1999, but not in the WIND list were observed during the period when WIND was inside the magnetosphere (25 March, 2122 April, 16 November). Mulligan et al. (1998) observed a steady increase in the yearly MC rate during the rising activity phase. They identified the largest number of MCs at solar maximum (1979) and in the declining phase (1982). Contrary to our study and the Wu et al. (2003) study, Lynch et al. (2003) identified the largest amount of MCs (20) in 2000 and in general the number of MCs is larger in their study. Almost $40 \%$ of all MCs in the Lynch et al. (2003) list are not included in our list. In comparison for the years 1997-2002 87\% of the MCs in the WIND list are included in our list. The differences between the studies are due to the different criteria to define MCs. For example, Lynch et al. (2003) have not limited the magnetic field total rotation to any specific value, whereas the total rotation of about $\sim 30^{\circ}$ is required in our study.

The comparison of Figs.3a and d indicates that the full and partial halo rate and the number of observed MCs at $1 \mathrm{AU}$ are not well correlated. For example, in 1997 LASCO observed only 19 halo CMEs and 15 partial halo CMEs compared to 61 halo CMEs and 100 partial halo CMEs observed in 2000. However, in 1997 more MCs were identified than in 2000.

Figure 4 presents the yearly distribution of MCs between left-handed and right-handed for the investigated period. In total, we found $42(58 \%)$ left-handed MCs and 31 (42\%) right-handed MCs.

\subsection{Solar cycle variation of the magnetic structure of MCs}

\subsubsection{Left- and right-handed MCs}

During 1999-2001 the left-handed MCs clearly outnumbered right-handed MCs. It is interesting to note that according to Table 2 during this period in all (13) identified $\mathrm{SN}$-type MCs magnetic field pointed east at the axis, i.e. they were left-handed. In 1997 and 2002 more right-handed MCs were observed than left-handed MCs. The relative number of left- and right-handed MCs obtained in this study is approximately in agreement with the previous studies: For 28 years of data Bothmer and Rust (1997) found that 52\% of
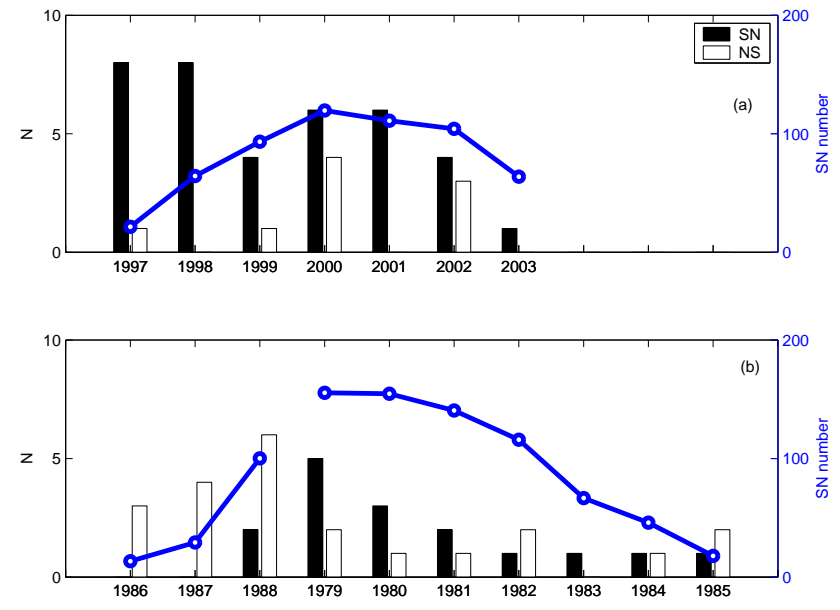

Fig. 5. Yearly number of MCs with magnetic field rotations from the south to the north (black) and from the north to the south (white) in our study (a) and in Mulligan et al. (1998) study (b). In (b) the years have been arranged to coincide with the years of approximately the same solar cycle phase in (a).

MCs were left-handed and 48\% right-handed. Bothmer and Schwenn (1998) also identified an almost equal distribution: $51 \%$ left-handed and 49\% right-handed MCs. In the set of MCs identified by Mulligan et al. (1998), 59\% were righthanded and $41 \%$ left-handed. For the three and one-half year period Lynch et al. (2003) found 55\% left-handed and 45\% right-handed MCs.

For the handedness of an MC there is no dependence on the solar cycle phase. The equal distribution between leftand right-handed MCs is expected over a time period of several years because generally left-handed MCs originate from the Northern Hemisphere and right-handed MCs from the Southern Hemisphere, Bothmer and Schwenn (1994); Rust (1994). This is based on the agreement of the field structure of MCs with the magnetic structure of the associated filament. Bothmer (2003) investigated in detail the solar sources of five MCs that are included in Table 2 (10-11 January 1997; 22 September 1997; 16-17 April 1999; 21-22 February 2000; $15-16$ July 2000). All of these five MCs followed the hemispheric rule. All front-side halo CMEs associated with these MCs originated from magnetic structures overlying polarity inversion lines and four of the five MCs were associated with disappearing $\mathrm{H}_{\alpha}$ filaments.

\subsubsection{SN vs. NS MCs}

The distribution of bipolar $\left(\theta_{C}<45^{\circ}\right) \mathrm{MCs}$ between those with the magnetic field rotation from the south to the north (SN) and from the north to the south (NS) in our study (a) and in the Mulligan et al. (1998) work (b) is displayed in Fig. 5. For the first three years of the investigated period (1997-1999) all bipolar MCs, except two (16 May 1997 and 18 February 1999) had southward fields in the leading part. The number of NS-type MCs increased during the last four years of the study: In 2000 we identified four and in 2002 
three NS-type MCs. The start of the change in the leading polarity of MCs at solar maximum was also observed by Bothmer and Rust (1997), Bothmer and Schwenn (1998) and Mulligan et al. (1998). As seen from Fig. 5b (note the arrangement of the years) during solar maximum and the declining phase of solar cycle 21 (1978-1984) both SN and NS type MCs were observed. The NS type MCs clearly dominated the SN type MCs from solar minimum to the next solar maximum (1985-1988).

\subsubsection{Bipolar vs. unipolar MCs}

Figures 6 and 7 display the changes in the axial inclination of the MCs as a function of time between 1997 and 2003. Figure 6 shows the variation of the absolute value of the inclination angle $\theta_{C}$ and Fig. 7 displays the yearly distribution between unipolar (i.e. $\left.\theta_{C}>45^{\circ}\right)$ and bipolar $\left(\theta_{C}<45^{\circ}\right) \mathrm{MCs}$ in our study (a) and in the Mulligan et al. (1998) work (b). MCs had a wide range of inclination angles $\left(1^{\circ}-78^{\circ}\right)$ and the scatter in Fig. 6 is large. The evolution of $\left|\theta_{C}\right|$ in time and the distribution of MCs between bipolar and unipolar in Fig. 7a reveal no systematic trend. We observed unipolar MCs frequently in the declining phase (2001 and 2003), but also during the rising activity phase (1997-1999) when each year about $40 \%$ of all identified MCs were unipolar. In 2000 and 2002 most MCs were bipolar. During the three years (1982-1984) of the late declining phase Mulligan et al. (1998) observed 13 unipolar MCs (70\%) compared to only four unipolar MCs $(21 \%)$ observed during the three years of the rising phase (1986-1988).

\subsection{Predicted travel times of MCs to $1 \mathrm{AU}$}

We studied carefully the LASCO and EIT images to find possible solar causes for each MC event at $1 \mathrm{AU}$. As the earthward coming CMEs appear as halos in the LASCO cororonagraph images their line-of-sight speed cannot be measured directly and arrival times to $1 \mathrm{AU}$ are hard to predict. For halo CMEs the radial speed is inaccessible, but the expansion speed can be determined. The method to determine the expansion speed is described in dal Lago et al. (2003) and Schwenn et al. (2005). Schwenn et al. (2005) measured $V_{\exp }$ for 75 LASCO CMEs which they were able to uniquely associate with shock waves in the SOHO, ACE or WIND solar wind data. For each CME-shock pair, the travel time $\left(T_{r}\right)$ to $1 \mathrm{AU}$ was determined. The function

$T_{t r}=203.0-20.77 \ln \left(V_{\exp }\right)$

fits the data best. In our study we found a unique CME association for $26 \mathrm{MCs}$ for which we were able to measure the expansion speed. We excluded many events that had a CME association, but for which the EIT images did not show clear front side activity. Also, in some cases there were multiple CME candidates in a sufficient time window or for a single $\mathrm{CME}$ no unique association at $1 \mathrm{AU}$ could be defined.

Figure 8 shows the travel times for MC leading edges (red stars) and for shocks (blue stars) plotted vs. the halo ex-

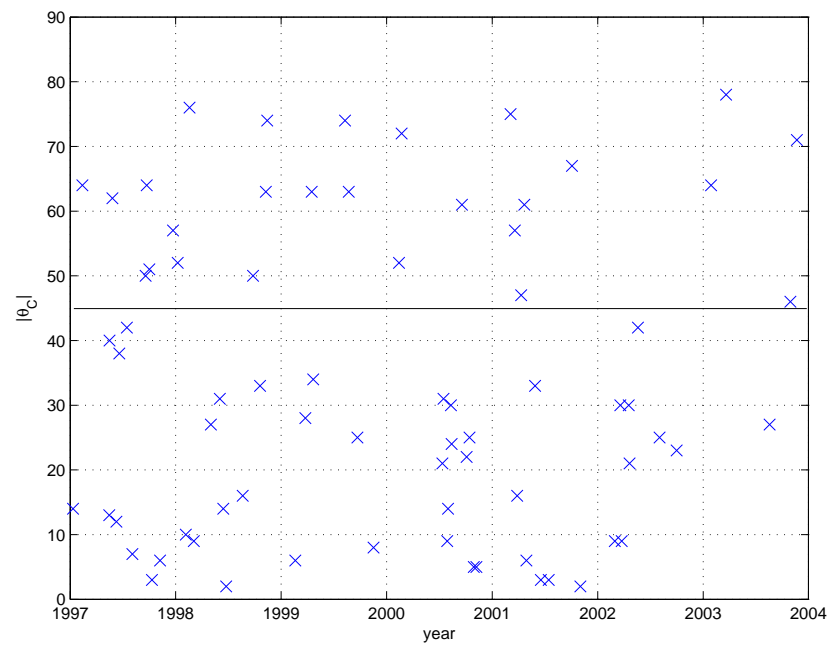

Fig. 6. Inclination angle $\theta_{C}$ with respect to the ecliptic plane.
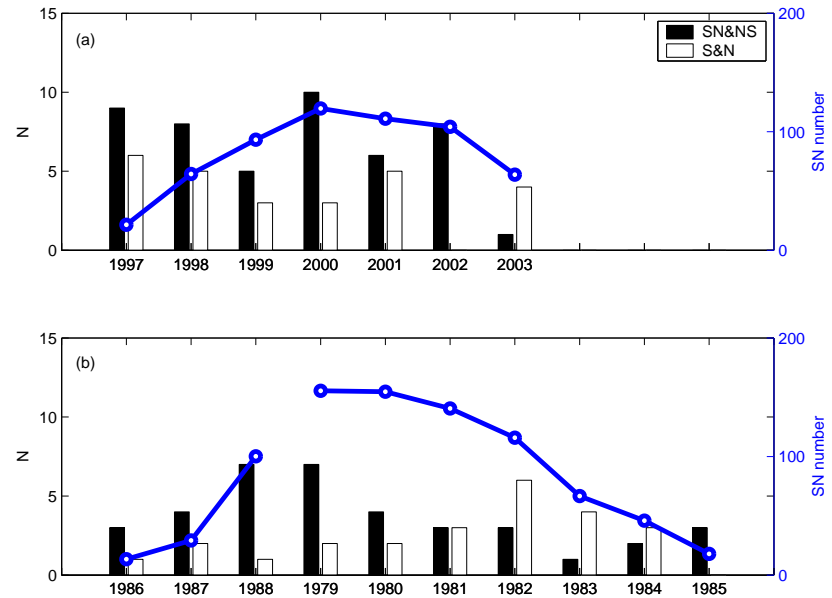

Fig. 7. Yearly number of bipolar (black) and unipolar (white) MCs in our study (a) and in Mulligan et al. (1998) (b). In (b) the years have been arranged to coincide with the years of approximately same solar cycle phase in (a).

pansion speed. The black dashed line indicates the calculated travel time from Eq. (2). A least-square fit curve of the same functional form as Eq. (2) but with newly derived coefficients using travel times of $25 \mathrm{MC}$ shocks in our study, $T_{t r}=236.7-25.94 \ln \left(V_{\exp }\right)$ is indicated by the blue line. The red line shows the same for CME-MC leading edge pairs, $T_{t r}=233.9-23.55 \ln \left(V_{\exp }\right)$. The standard deviation is $11.4 \mathrm{~h}$ for $26 \mathrm{CME}-\mathrm{MC}$ leading edge pairs, and 9.66 for $25 \mathrm{CME}-$ shock pairs in our study, while for the 75 shocks in ? it was $14 \mathrm{~h}$. The scatter in Fig. 8 is still substantial. One would expect to find an improvement when the travel time of the MC leading edge or shocks is used instead of the travel time of all the uniquely CME associated shocks at $1 \mathrm{AU}$. A shock is a larger scale structure than the CME driving it (Sheeley, 1985). When the shock-CME ejecta structure is cut at the flanks where CME material is not present, $T_{t r}$ is increased 


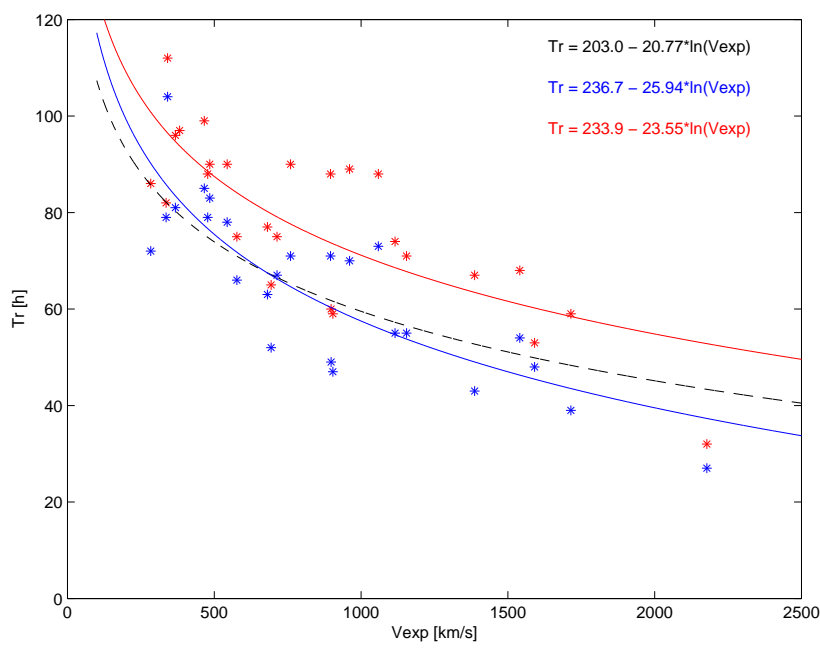

Fig. 8. Travel times for shocks (blue stars) and MC leading edges (red stars) vs. halo expansion speed. The black dashed line gives the least-squares fit for 75 CME-shock pairs in Schwenn et al. (2005). Blue and red lines give the least-squares fit for $26 \mathrm{CME}$-shock and CME-MC leading edge pairs in this study.

relative to the shock-CME structure that is cut near the center. In this study we have correlated halo CMEs to MCs. Thus, for all events the structure is cut relatively close to the center (as otherwise they would not have been identified MCs at all).

\section{Geoeffectiveness of MCs}

The geoeffectiveness of the identified as MCs was examined using the $1-\mathrm{h} D_{s t}$ index. Final values of $D_{s t}$ were available for 1997-2002 and preliminary values were used for 2003. In the figures presented in this section we also give the pressure corrected $D_{s t}\left(D_{s t}^{*}\right)$, where the contribution of the magnetopause currents have been removed by using the equation in Burton et al. (1975):

$D_{s t}^{*}=D_{s t}-b \sqrt{P_{\mathrm{dyn}}}+c$,

where $P_{\text {dyn }}$ is the solar wind dynamic pressure and for constants $b$ and $c$ we have used values $b=7.26 \mathrm{nT}(\mathrm{nPa})^{1 / 2}$ and $c=11 \mathrm{nT}$ derived by O'Brien and McPherron (200a). Following the classification by Gonzalez et al. (1994) we defined moderate storms to have their $D_{s t}$ minimum between $-50 \mathrm{nT}$ and $-100 \mathrm{nT}$ and intense storms to have the $D_{s t} \mathrm{~min}-$ imum $<-100 \mathrm{nT}$. We have taken into consideration whether the storm was caused by southward fields embedded in the MC part itself or by sheath fields. We defined the cause of the storm as the structure (i.e. sheath or MC) during which $D_{s t}$ reached $85 \%$ of its minimum for that particular storm. Column 12 in Table 2 shows the $D_{s t}$ minimum (if it is less than $-50 \mathrm{nT}$ ) for each MC. If the sheath caused the storm, we have indicated it by "sh" and the $D_{s t}$ minimum follows in parentheses. We have excluded an event (9 June 1997) that occurred in the recovery phase of the previous storm, as the contribution of the MC fields to the $D_{s t}$ behavior was not clear. When $D_{s t}$ had more than one depression before attaining its minimum value, we used the definition described by Kamide et al. (1998) to determine whether the event was interpreted as a two-step magnetic storm or two separate magnetic storms: Assume that the magnitude of the first $D_{s t}$ depression is $A$ and $D_{s t}$ recovers by an amount $C$ before the second depression. If $C / A>0.9$, the $D_{s t}$ decreases are classified as two separate magnetic storms.

Gonzalez et al. (1994) presented solar wind threshold values for moderate and intense storms: A moderate storm is generated when $B_{z}$ is less than $-5 \mathrm{nT}$ for more than $2 \mathrm{~h}$, and intense storms are caused by a $B_{z}$ less than $-10 \mathrm{nT}$ lasting more than $3 \mathrm{~h}$. Gonzalez and Tsurutani (1987) also required that in order for an intense storm to be generated the solar wind electric field $\left(E_{s w}\right)$ should be larger than $5 \mathrm{mV}$ at least for $3 \mathrm{~h}$ concurrently with $B_{z}<-10 \mathrm{nT}$.

\subsection{MCs without storms}

For 21 events out of a total of 72 neither the sheath nor the MC caused the $D_{s t}$ decrease below our storm limit. The majority of the 21 MCs that did not cause a storm had low magnetic field intensity or were $\mathrm{N}$-type with no significant southward fields in the sheath. The average peak of the magnetic field magnitude of all $73 \mathrm{MCs}$ in our study was $18.6 \mathrm{nT}$ and the average of the maximum speed inside an MC was $477 \mathrm{~km} / \mathrm{s}$ (for $70 \mathrm{MCs}$, as three events lacked solar wind measurements). The average peak magnetic field for the 20 nongeoeffective MCs was only $13.2 \mathrm{nT}$ and the average speed was slightly less than that for all MCs, $463 \mathrm{~km} / \mathrm{s}$. An example of a non-geoeffective ENW-type MC on 22 September 1997 has been presented by Bothmer (2003).

Three events from these 21 cases fulfilled the Gonzalez et al. (1994) threshold for a moderate storm: 15-16 July 1997; 3-4 August 1997 and 25 March 1999. The solar wind measurements from WIND and the geomagnetic response for the MC on 3-4 August 1997 are shown in Fig. 9. The figures show the magnetic field intensity, $B_{z}$ component (in the GSM coordinate system), solar wind electric field, dynamic pressure, and the $D_{s t}$ index (solid line) with the pressure correction (dashed line). The data have not been shifted to the magnetopause. WIND was located at the GSE position of $(X, Y, Z)=(80,70,12) R_{E}$ and the time delay from WIND to the magnetopause was about $20 \mathrm{~min}$. The leading edge of the MC arrived at WIND at 14:00 UT on 3 August. Within the MC the magnetic field vector rotated from the south to the north. The magnetic field $Z$-component was less than $-10 \mathrm{nT}$ (with a minimum value $-13 \mathrm{nT}$ ) for more than $4 \mathrm{~h}$, with concurrently $E_{s w}$ larger than $5 \mathrm{mV} / \mathrm{m}$ for about three and one-half hours. This event even met the criteria for an intense magnetic storm, but $D_{s t}$ decreased only to $-49 \mathrm{nT}$ (the $D_{s t}^{*}$ minimum also $-49 \mathrm{nT}$ ). 


\subsection{Sheath storms}

In 16 cases the $D_{s t}$ minimum of the storm was caused by sheath fields preceding the MC. In six cases the following $\mathrm{MC}$ had southward fields in the leading part. The SN-type MC observed on 15-16 May 1997 had a $B_{z}$ less than - 10 nT for about three and one-half hours, with the minimum value of $-24 \mathrm{nT}$. This MC would have been geoeffective itself, but during the sheath $D_{s t}$ decreased to $-100 \mathrm{nT}$, that is $87 \%$ of the storm $D_{s t}$ minimum of $-115 \mathrm{nT}$ that was reached only four hours later. Thus, this was classified as a sheath storm according to our definition. However, the contribution of the magnetopause currents to $D_{s t}$ was larger during the sheath than during the $\mathrm{MC}$, and the pressure corrected $D_{s t}$ reached its minimum already during the sheath (Liemohn et al, 2001). MCs whose sheath region caused a storm had an average peak magnetic field magnitude of $16.6 \mathrm{nT}$ (slightly less than the average value of all MCs). The average of the maximum speed was $519 \mathrm{~km} / \mathrm{s}$, that is above the average for all MCs. This is as expected, as the draping of the ambient interplanetary magnetic field about the CME in the sheath is more efficient the larger the CME speed is relative to the ambient plasma (Gosling and McComas, 1987).

Figure 10 shows an example of an SN-type MC on 6-7 November 2000 whose sheath region caused an intense magnetic storm. The shock was observed at ACE on 6 November at 09:08 UT. ACE is located near the L1 point $\sim 220 R_{E}$ from the Earth so the time delay from ACE to the magnetopause was about $40 \mathrm{~min}$. In the sheath the IMF was mainly southward and caused the $D_{s t}$ decrease to $-159 \mathrm{nT}\left(D_{s t}^{*}-172 \mathrm{nT}\right)$ on 6 November at 22:00 UT. The $D_{s t}$ minimum was clearly caused by the sheath fields as the front edge of the MC reached the magnetopause on 6 November at 23:00 UT. In the end of the sheath region the IMF turned northward and $D_{s t}$ started to recover. A few hours later southward fields in the leading part of the MC caused a second depression of $D_{s t}$. Before the $D_{s t}$ minimum in the sheath $B_{z}$ was less than $-10 \mathrm{nT}$ for nearly four hours with the minimum value at $-13 \mathrm{nT}$.

It is interesting to compare the interplanetary conditions and geomagnetic responses between the events presented in Figs. 9 and 10. The magnitude and duration of southward $B_{z}$ before the $D_{s t}$ minimum were comparable between these two events. The solar wind speed was somewhat higher during the 6-7 November 2000 sheath than during the 3-4 August $1997 \mathrm{MC}$, and the maximum of the solar wind electric field were $8 \mathrm{mV} / \mathrm{m}$ and $6.5 \mathrm{mV} / \mathrm{m}$, respectively. It seems quite peculiar why the $B_{z}$ conditions shown in Fig. 10 led to an intense magnetic storm while those presented in Fig. 9 did not cause a storm at all. During southward IMF for the 3-4 August 1997 event the dynamic pressure was low $(\sim 2 \mathrm{nPa})$ while for the 6-7 November 2000 event the dynamic pressure was up to $15 \mathrm{nPa}$. The relative change in $D_{s t}^{*}$ was $62 \mathrm{nT}$ for the 3-4 August 1997 event and 143 nT for the 6-7 November 2000 storm.

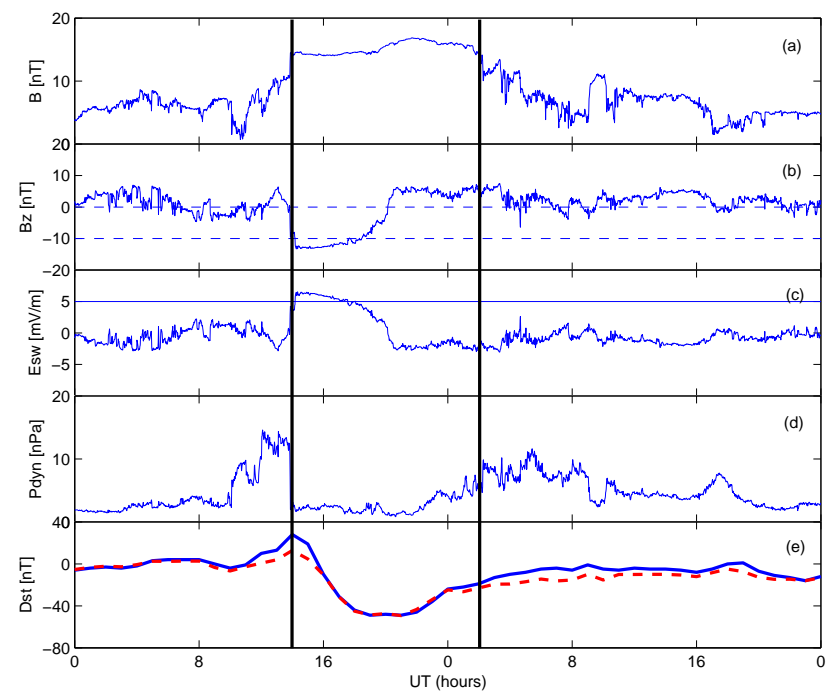

Fig. 9. Solar wind parameters and geomagnetic indices for a 2-day interval from 3-4 August 1997 measured by WIND. The figures from top to bottom show magnetic field strength (a), magnetic field $B_{z}$-component in the GSM coordinate system (b), solar wind dynamic pressure $(\mathbf{c})$, solar wind electric field (d) and the $D_{s t}$ index (solid line) together with the pressure corrected $D_{s t}$ (dashed line) (e). Two solid lines indicate the interval of an MC.

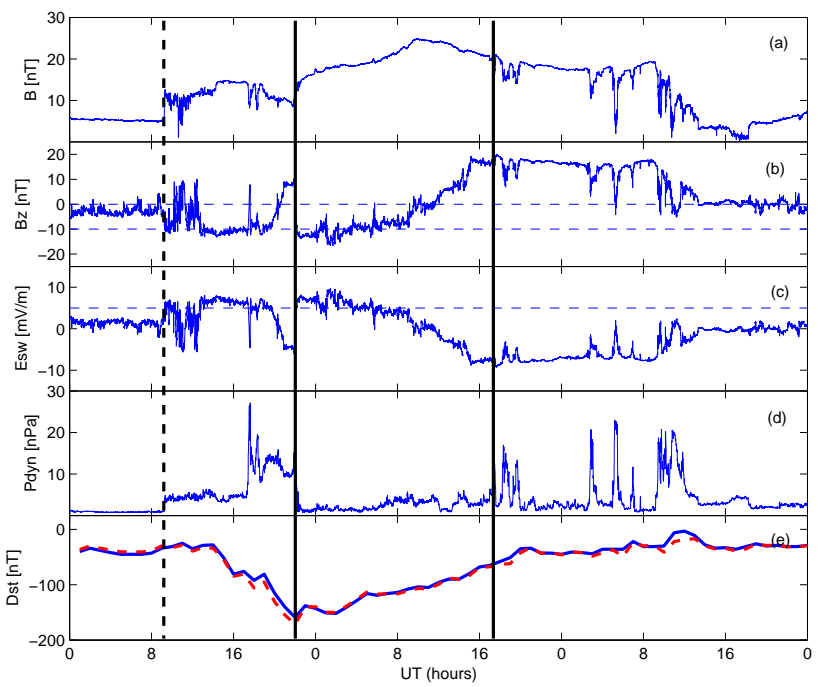

Fig. 10. Solar wind parameters and geomagnetic indices for a 3-day interval from 6-8 November 2000 measured by ACE. The figures from top to bottom are the same as in Fig. 9. The dashed line indicates the shock and two solid lines indicate the interval of an MC.

\subsection{Moderate and intense storms}

Southward fields within the MC part itself caused 15 moderate storms and 20 intense storms. On the average the geoeffective MCs had a larger peak magnetic field magnitude $(21.7 \mathrm{nT})$ and the speed was of the same order as the average of all MCs $(472 \mathrm{~km} / \mathrm{s})$. MCs on 15-16 July 2000 and 29-30 October 2003 that caused major magnetic storms and 


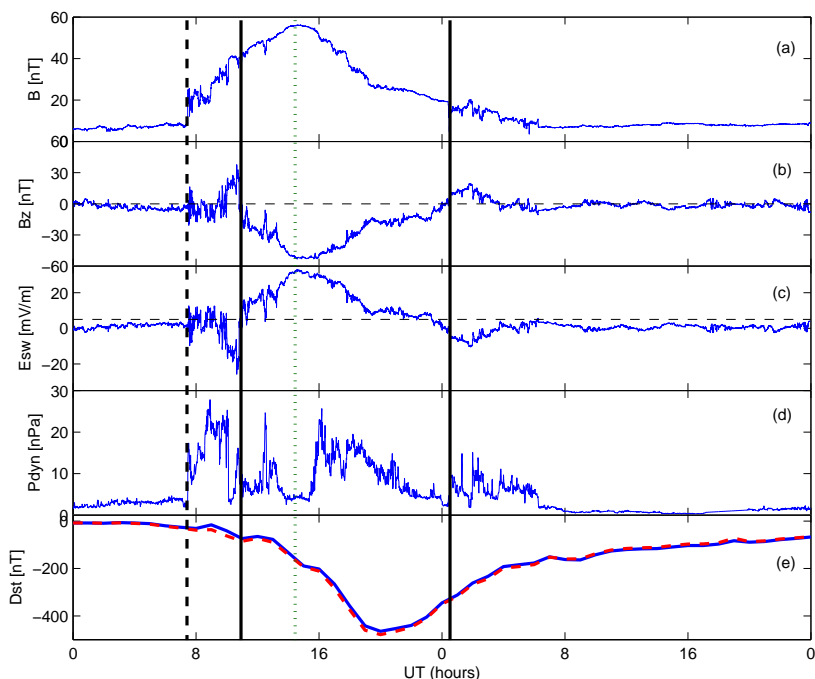

Fig. 11. Solar wind parameters and geomagnetic indices for a 2day interval from 20-21 November 2003 measured by ACE. The figures from top to bottom are the same as in Fig. 9. The dashed line indicates the shock and two solid lines indicate the interval of the MC.

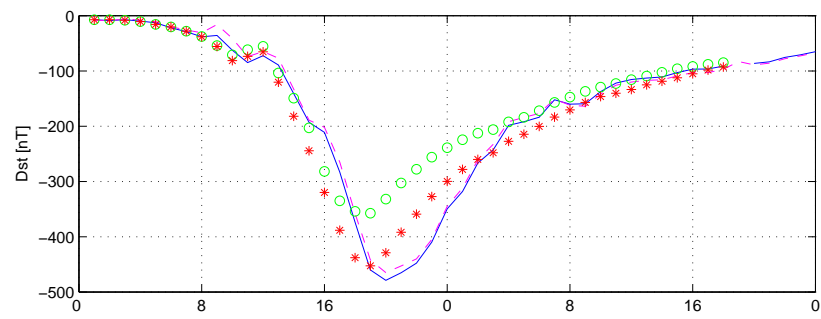

Fig. 12. Measured and predicted $D_{s t}$ development for 20-21 November 2003. The blue solid line is the 1-h $D_{s t}$ index and the purple dashed line is $D_{s t}^{*}$. The green open circles show the predicted $D_{s t}^{*}$ using the O'Brien and McPherron (2000a) model and the red stars the predicted $D_{s t}^{*}$ using the Wang et al. (2003a) model.

had very intense magnetic fields, lacked solar wind measurements.

The MC on 10-11 January 1997, see Bothmer (2003), caused only a moderate storm $\left(D_{s t}\right.$ minimum $\left.-78 \mathrm{nT}\right)$, although $B_{z}$ had values less than $-10 \mathrm{nT}$ (with the minimum value $-15 \mathrm{nT}$ ) for four and one-half hours, and $E_{s w}$ was larger than $5 \mathrm{mV} / \mathrm{m}$ for more than six hours. The dynamic pressure was low (2-4 nPa) during southward IMF. The 1617 April 1999 MC, see Bothmer (2003) and also the 16 November $1999 \mathrm{MC}$ had $B_{z}$ less than $-10 \mathrm{nT}$ longer than $3 \mathrm{~h}$. During the 16-17 April 1999 event $E_{s w}$ was larger than $5 \mathrm{mV}$ for two and one-half hours and the 16 November 1999 event lacked solar wind measurements. They both caused moderate storms $(-91 \mathrm{nT}$ and $-79 \mathrm{nT})$.

It was shown by Huttunen and Koskinen (2004) that sheath regions were the most important drivers of intense magnetic storms during the period 1997-2002. However, three of the four most intense magnetic storms associated with the
$D_{s t}$ decrease below $-300 \mathrm{nT}$ during the solar cycle 23 were driven primarily by southward fields in an MC. These storms were the "Bastille Day" storm on 15-16 July 2003, the first of the "Halloween storms" on 29-30 October 2003 (the second Halloween storm on 31 October 2003 was presumably driven by sheath fields) and the storm on 20-21 November 2003. This is understandable because only within MCs the southward magnetic field can obtain highest intensities.

\subsubsection{0-21 November 2003 storm}

Figure 11 shows an example of the intense magnetic storm on 20-21 November 2003 that was driven by southward fields in MC. When defined by $D_{s t}$ this was the most intense magnetic storm during the solar cycle 23. An interplanetary shock was observed at ACE on 20 November at 07:27 UT. In the sheath the magnetic field fluctuated from the south to the north and initiated the $D_{s t}$ decrease below $-50 \mathrm{nT}$. A very well-defined MC arrived at ACE on 20 November at 11:00 UT. The calculated orientation of the MC's axis was $\left(\phi_{C}, \theta_{C}\right)=\left(40^{\circ}, 71^{\circ}\right)$. The $\mathrm{MC}$ can be classified as the flux-rope category ESW and the variation in the magnetic field was right-handed. The magnetic field $Z$-component was southward during the whole passage of the $\mathrm{MC}$ and the maximum of the magnetic field coincided approximately with the minimum value of $B_{z}$. The magnetic field magnitude was exceptionally high, almost $60 \mathrm{nT}$, and the minimum value of $B_{z}$, was $-53 \mathrm{nT}$, was reached at 15:12 UT on 20 November, after which the magnetic field vector rotated slowly back to zero. Solar wind dynamic pressure was high inside the MC. Southward MC fields caused most of the $D_{s t}$ decrease and the minimum value of $D_{s t},-465 \mathrm{nT}\left(D_{s t}^{*}-479 \mathrm{nT}\right)$, was reached at 20:00 UT on 20 November.

Figure 12 shows the predicted $D_{s t}^{*}$ development according to the O'Brien and McPherron (2000a) and Wang et al. (2003a) models. The O'Brien and McPherron (2000a) model assumes that the ring current injection and ring current decay parameter are controlled by the solar wind electric field. The Wang et al. (2003a) model is a modification of the O'Brien and McPherron (2000a) model and includes the influence of the solar wind dynamic pressure in the injection function and the decay parameter. Wang et al. (2003a) predicts notably well the magnitude of the $D_{s t}^{*}$ minimum while the O'Brien and McPherron (2000a) model clearly underestimates the $D_{s t}^{*}$ minimum (the O'Brien and McPherron (2000a) model is adjusted to $D_{s t}>-150 \mathrm{nT}$ ). Thus, it seems that for this storm the solar wind dynamic pressure had an important contribution to the ring current development. This is also seen from Fig. 11 as $D_{s t}$ was further depressed by about $200 \mathrm{nT}$ after the magnetic field had turned less southward and the dynamic pressure was increased to about $20 \mathrm{nPa}$.

The MC on 20-21 November was most probably caused by a halo CME detected in LASCO images on 18 November 2003. The CME was first detected at the LASCO C2 field of view at 08:50 UT. EIT images showed activity almost at the center of the solar disk. Two M-class flares (M3.2 and M3.9) occurred in the active region 501, located almost at the center 


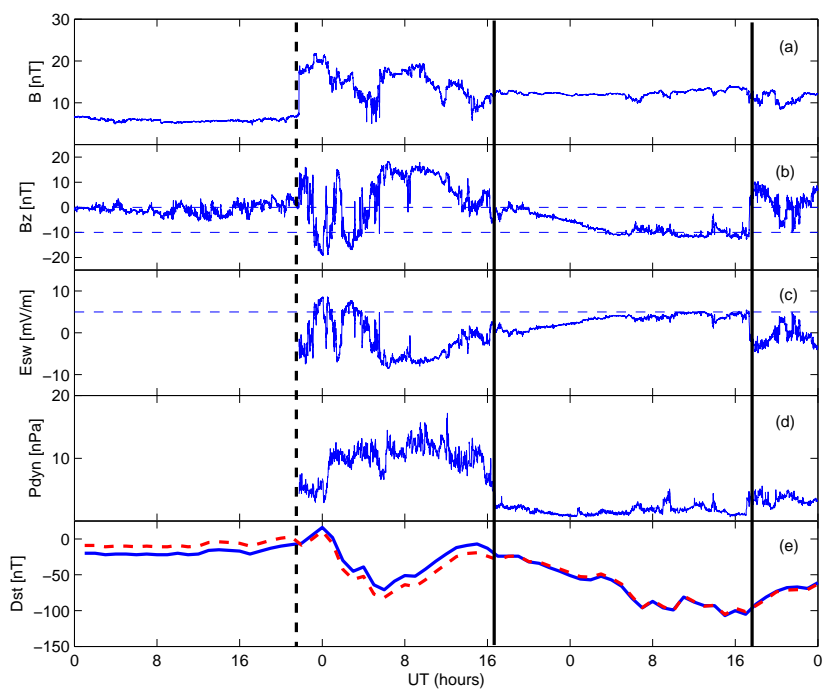

Fig. 13. Solar wind parameters and geomagnetic indices for a 3-day interval from 12-14 October 2000 measured by ACE. The figures from top to bottom are the same as in Fig. 9.

of the solar disk (N00E18) at 07:52 UT and 08:31 UT. Additionally, $\mathrm{H}_{\alpha}$ images show a disappearance of a large filament structure south of the active region.

\subsubsection{Main phase development}

Kamide et al. (1998) suggested that the two-step development of $D_{s t}$ that is present for more than $50 \%$ of intense storms can be caused when southward $B_{z}$ fields are present both in the sheath and in the MC. For SN-type MCs the average time difference between the $D_{s t}$ peaks was small (7 h) because of the close spatial proximity of the sheath fields and the southward $B_{z}$ in the MC.

For NS-type MCs the separation between southward $B_{z}$ fields in the sheath and in the MC can be so large that $D_{s t}$ has enough time to recover to non-storm values and two separate magnetic storms follow. Figure 13 shows an NS-type MC that was observed by ACE during 13-14 October 2000. The shock arrived at ACE at 21:36 UT on 12 October. The sheath caused a moderate storm with the $D_{s t}$ minimum $-71 \mathrm{nT}$ ( $D_{s t}^{*}$ $-81 \mathrm{nT}$ ) on 13 October, 06:00 UT. The southward $B_{z}$ in the trailing part of the MC caused an intense storm, with the $D_{s t}$ minimum was $-107 \mathrm{nT}\left(D_{s t}^{*}-105 \mathrm{nT}\right)$ on 14 October 15:00 UT. The time difference between the two $D_{s t}$ minima was $34 \mathrm{~h}$.

Another NS-type MC that caused two separate magnetic storms occurred on 28-29 July 2000. The storm caused by the sheath had the $D_{s t}$ minimum of $-51 \mathrm{nT}\left(D_{s t}^{*}-60 \mathrm{nT}\right)$ and $27 \mathrm{~h}$ later the MC caused a $D_{s t}$ minimum of $-71 \mathrm{nT}$ $\left(D_{s t}^{*}-79 \mathrm{nT}\right)$. From the remaining seven identified NS-type MCs, one caused an intense storm (30 September - 1 August 2002), but there was no significant southward $B_{z}$ in the sheath; four mcs were not geoeffective at all and in two cases only sheath fields caused the storm.

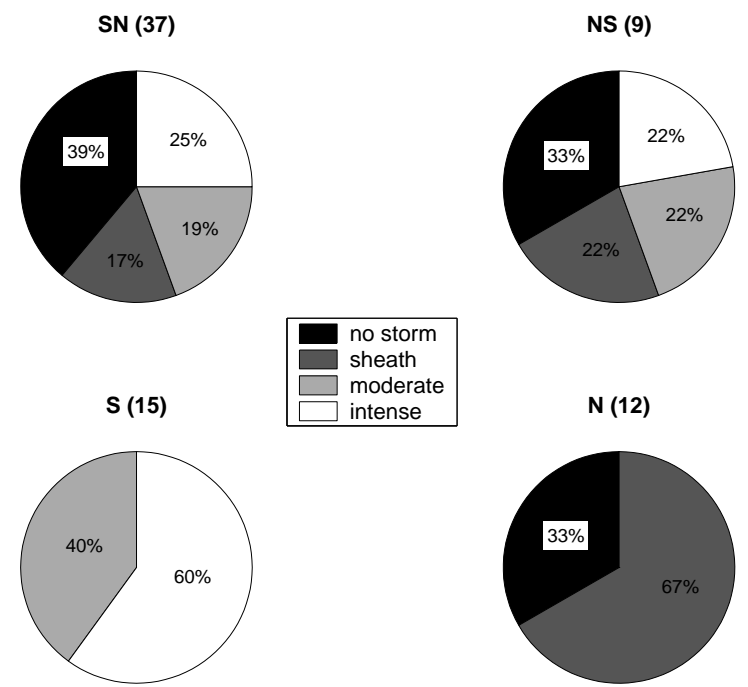

Fig. 14. The effect of the flux rope type to the geoeffectivity. Numbers in the parentheses show the total numbers of MCs identified in each category. Different colors demonstrate the different geomagnetic response: no storm at all, $D_{s t}>-50 \mathrm{nT}$ (black); sheath region generated a storm (dark gray); MC caused a moderate storm (light gray); MC caused an intense storm (white).

\subsection{Geomagnetic response of MCs with different flux rope types}

Figure 14 summarizes the geomagnetic response of MCs belonging to different flux rope categories. The pie-diagrams in the top part of the figure show the distribution for bipolar MCs. In more than half of the events either the sheath region caused the storm or no significant activity at all was generated. It is interesting to note that when geoeffective, the SN type MCs caused more intense storms than moderate storms.

For bipolar MCs the respond depends clearly on the direction of the magnetic field on the axis. In total we identified 15 S-type MCs. As seen from Fig. 14 all of them caused a storm: nine caused an intense storm (23 November 1997; 18 February 1998; 9 November 1998; 13 November 1998; 20 March 2001; 22 April 2001; 3 October 2001; 30 October 2003; 20 November 2003) and six caused a moderate storm (27 May 1999; 17 April 1999; 23 August 1999; 5 March 2001; 29 February 2002; 20 March 2003).

From $12 \mathrm{~N}$-type MCs none caused a storm. However, for eight $\mathrm{N}$-type MCs the sheath region preceding the MC generated a storm. Half of these were intense magnetic storms. For example, the sheath preceding the N-type MC on 25-26 September 1998 caused an intense magnetic storm with the $D_{s t}$ minimum $-207 \mathrm{nT}$.

\section{Discussion}

We have investigated the properties of 73 MCs identified from WIND and ACE measurements during 1997-2003, covering rising, maximum and early declining phases of so- 
lar cycle 23. The investigated period does not cover the whole solar cycle 23 , but we have almost continuous coverage of solar wind measurements. We applied the minimum variance analysis to determine whether the preselected candidate $\mathrm{MC}$ regions exhibited smooth rotation of the magnetic field in one plane. We also required that MCs must be lowbeta structures (averages values of beta within the MC less than 0.5 ) with the maximum magnetic field magnitude $8 \mathrm{nT}$ or larger and the duration at least $6 \mathrm{~h}$.

We identified the largest number of MCs during the early rising phase when the solar activity was still low (19971998). The number of observed MCs dropped in 1999, but increased again at solar maximum (2000). After that the MC rate started to decrease with the declining solar activity. The number of MCs observed at $1 \mathrm{AU}$ did not correlate with the number of wide (angular width $>120^{\circ}$ ) LASCO CMEs. Cane and Richardson (2003) found that near solar minimum nearly $100 \%$ of all observed ICMEs at $1 \mathrm{AU}$ had the MC structure and the fraction decreased to $10-20 \%$ when solar maximum was reached.

The occurrence rate of MCs is naturally affected by the criteria used to define an MC. In general, MCs are easier to identify from the solar wind near solar minimum than solar maximum. Near solar maximum the mutual interaction between CMEs and the ambient solar wind can lead to complex structures at $1 \mathrm{AU}$ where the individual characteristics of CME(s) are no longer visible, Gopalswamy et al. (2001); Burlaga et al. (2001); Wang et al. (2003b). A large fraction of MCs can be associated with disappearing filaments, Wilson and Hildner (1986); Bothmer and Schwenn (1994); Bothmer and Rust (1997) and it is likely that CMEs originating from the active regions rarely have an MC structure. Filaments drift towards poles when solar activity increases, contrary to the sunspots and active regions that migrate towards the equator (Hundhausen, 1993). Near solar minimum there are few active regions and the filament disappearances occur close to the equator. Furthermore, it has been shown that near solar minimum CMEs are systematically deflected equatorward by the fast solar wind flow originating from large polar coronal holes (Cremades and Bothmer, 2004). This suggests that most solar minimum CMEs have an MC structure and when encountering the Earth they are crossed near the axis. Near solar maximum the filament eruptions occur mainly at high latitudes and the number of CMEs are not deflected at all or are deflected towards the poles (Cremades and Bothmer, 2004). As a consequence, MCs arising from these filament sites miss the Earth completely or are crossed far from the axis. The earthward-directed CMEs that mainly originate from the active regions near the equator do not generally have the MC structure. Wu et al. (2003) pointed out that the low number of MCs observed in 1999 was likely due to the fact that most filament disappearances occurred at very high latitudes this year. The total number of MCs that encountered the Earth during the solar maximum years was likely larger than reported in Table 2, but our criteria did not identify these as MCs. Also, it should be noted that although we could reliably identify all MCs at $1 \mathrm{AU}$, we could not necessarily draw conclusions about the total number of MCs expelled from the Sun, as an increasingly larger amount of MCs are expelled from higher latitudes never reaching the Earth when solar maximum is approach.

We identified somewhat more left-handed than righthanded MCs (58\% and 42\%). Also, in the previous studies the total amount of left-handed MCs was slightly larger than the total amount of right-handed MCs. The equal amount of left-handed and right-handed MCs is expected over the time interval of several years, as left-handed MCs originate from the Northern Hemisphere and right-handed MCs from the Southern Hemisphere, Bothmer and Schwenn (1994); Rust (1994). The largest difference was observed during the years of high solar activity (1999-2001) when the magnetic equator of the Sun is not as well defined as near solar minimum.

From minimum variance analysis we obtained the estimation for the orientation of the MC axes that we used to separate MCs from those lying near the ecliptic plane (bipolar, $\theta_{C}<45^{\circ}$ ) and those perpendicular to the ecliptic plane (unipolar, $\theta_{C}>45^{\circ}$ ). In total we identified 46 bipolar MCs (63\% from all MCs). During the rising phase nearly all identified bipolar MCs were of the type SN. At solar maximum and in the declining phase several NS-type MCs were observed.

Figure 18 in Bothmer and Rust (1997) demonstrates how the magnetic structures of filaments and overlying magnetic arcades are associated with the flux rope types of MCs and their solar cycle changes. The suggested pre-eruptive configuration of MCs consists of large-scale magnetic field arcades overlying neutral lines/filament sites in bipolar regions, e.g. Gosling et al. (1995); Martin and McAllister (1997). The number of bipolar regions increases clearly when the solar activity is high and the pre-eruption field configuration may also form between two neighboring bipolar regions, Tandberg-Hanssen (1974); Tripathi et al. (2003). MCs originating from the magnetic field configuration connecting two bipolar regions would have a different sense of rotation than those forming from a single bipolar region. Furthermore, both NS- and SN-type MCs are observed during the periods when magnetic regions from both the old and the new cycle are present, i.e. during the declining activity cycle. In the minimum and rising activity phases, when only a few bipolar regions from a single cycle are present, the majority of MCs have the same sense of magnetic field rotation.

In total, we found 23 unipolar MCs (37\%). Mulligan et al. (1998) suggested that the orientation of the coronal streamer belt controls the inclination angle of the MC axis. They interpreted their results that unipolar MCs are most frequent in the declining phase when the neutral line is in many regions tilted at large angles to the solar equator, while during solar minimum and the rising phase, when the streamer belt is more equatorial, MCs are mainly bipolar (Hoeksema, 1995). This is not consistent with our study, as we frequently observed unipolar MCs in the rising phase, where the fraction of unipolar MCs was about $40 \%$ for each year, while at maximum and in the declining phase the fraction varied from 0 to $80 \%$. We found no clear and systematic trend in the axial 
orientation of MCs with respect to the ecliptic. Marubashi (1997) and Zhao and Hoeksema (1998) have demonstrated that the orientation of the $\mathrm{MC}$ axis relative to the ecliptic plane correlates rather well with the tilt of the associated filament relative to the solar equator. For filaments studied by Cremades and Bothmer (2004) between 1996 and 2002 no systematic trend was observed in the tilt, but a tendency for low inclined cases was observed after 2000. Apparently, the deflection of CMEs by the ambient coronal solar wind flow can deviate the CME axis from the associated filament orientation (Cremades and Bothmer, 2004).

The geomagnetic response of MCs was investigated using the 1-h $D_{s t}$ index. We focused on whether the storm was caused by sheath fields or by the MC itself. Sheath regions are often associated with a fluctuating IMF direction and high dynamic pressure while MCs have a smoothly changing IMF direction and low dynamic pressure. Thus, they put the magnetosphere under very different solar wind input, (Huttunen et al. (2002a); Huttunen and Koskinen (2004). About onethird of MCs that encounter the Earth do not cause a storm at all (when defined as $D_{s t}<-50 \mathrm{nT}$ ). These MCs are typically somewhat slower and have lower magnetic field magnitudes than the average $\mathrm{MC}$ at $1 \mathrm{AU}$. We found that a sheath region caused a storm in almost one-fourth of the cases. Thus, in half of the events the southward $B_{z}$ embedded in the MC was the primary cause of the storm. MCs are inclined to cause intense magnetic storms since out of 35 storms caused by MCs, 20 had a $D_{s t}$ below -100 nT. However, six MCs that met the solar wind threshold criteria for moderate or intense storms, Gonzalez et al. (1994), had a $D_{s t}$ response less intense than expected. Tsurutani et al. (2003) investigated ring current intensification during 11 storm main phases in 1997 that were caused by a smoothly varying $B_{z}$ component within MCs. In 5 cases they found a lack of substorm expansion phase for a long period which they suggested to be the cause of the low intensity of the storm.

The geomagnetic response of an MC depends greatly on its flux-rope type. For the S-type MC the magnetic field is purely southward at the axis where the magnetic field has its maximum value, see Eq. (1). All 15 identified S-type MCs caused a storm, nine of them an intense storm (e.g. the largest storm of the solar cycle 23 on 19-20 November 2003). On the contrary, from the 12 identified $\mathrm{N}$-type MCs none caused a storm, but for eight of these MCs the sheath region preceding the MC itself was geoeffective. There are still large uncertainties in determining the travel time of the CMEs from the Sun to the Earth (?). We investigated the relation between the travel time of the MC shock and the leading edge to $1 \mathrm{AU}$ and the expansion speed of the associated halo CME. The results were slightly better in comparison to ?, who investigated the relationship between expansion speed and all halo $\mathrm{CME}$ associated shocks at $1 \mathrm{AU}$.

\section{Summary}

The magnetic structure and geomagnetic response of MCs detected by the WIND and ACE satellites are investigated during solar cycle 23 . The results confirm the solar cycle evolution in the leading polarity of MCs found for the previous cycles (21-22) by Bothmer and Rust (1997), Bothmer and Schwenn (1998) and Mulligan et al. (1998), but we did not find a clear and systematic trend in the axial inclination of MCs with respect to the ecliptic. MCs that are highly-inclined ("unipolar") were frequently observed almost throughout the time investigated. This result is important for the predictive purposes, as unipolar MCs that have the field southward at the axis are particularly geoeffective. In the rising phase nearly all "bipolar" MCs that are lying near the ecliptic plane were associated with the SN rotation. At solar maximum and in the declining phase the number of bipolar MCs with the opposite sense of rotation was increased. We suggest that at solar maximum the grouping of bipolar regions and in the declining phase the presence of magnetic regions from both new and old solar cycles, results in the mixture of NS and SN type MCs.

The geomagnetic response of MCs varied greatly depending on the inferred flux-rope category. When geoeffective, the MCs have a tendency to cause intense magnetic storms. By distinguishing the contribution of the sheath region and the $\mathrm{MC}$ itself we find that in the considerable fraction of cases $(22 \%)$ the sheath region caused the $D_{s t}$ minimum of the storm. In particular, the intensity and duration of southward $B_{z}$ in the sheath is crucial for N-type MCs, as they are not geoeffective themselves. In principle, the flux-rope type of an MC can be deduced in advance from the magnetic structure of the associated filament, e.g. Bothmer and Schwenn (1998), but for the sheath fields no practical method has been developed. Another important aspect is to reliably predict the time of the storm. As shown in this study, there are still large uncertainties in determining the MC arrival time from the Sun to $1 \mathrm{AU}$. Whether the storm is caused by the southward $B_{z}$ values in the sheath, in the leading part of the MC or in the trailing part of the $\mathrm{MC}$, can make a large difference as to the timing of the storm. Particularly, an NS-type MC may cause two separate magnetic storms due to a long separation of southward fields in the sheath and in the MC between.

Acknowledgements. We thank R. Lepping for the WIND magnetic field data, and K. W. Ogilvie for the WIND solar wind data. We also thank N. Ness for the ACE magnetic field data and, D. J. McComas for the ACE solar wind data. These data were obtained through Coordinated Data Analysis Web (CDAWeb). The LASCO CME catalog is generated and maintained by NASA and The Catholic University of America in cooperation with the Naval Research Laboratory. SOHO is a project of international cooperation between ESA and NASA. The yearly sunspot numbers were obtained from RWC Belgium World Data Center for the Sunspot Index. The $D_{s t}$ and $K_{p}$ values were obtained from the World Data Center C2 in Kyoto. The study was supported through the Antares programme of the Academy of Finland.

Topical Editor R. Forsyth thanks C. Cid and another referee for their help in evaluating this paper. 


\section{References}

Bame, S. J., Asbridge, J. R., Feldman, W. C., Gosling, J. T., and Zwickl, R. D.: Bi-directional streaming of solar wind electrons $>80 \mathrm{eV}$ : ISEE evidence for a closed-field structure within the driver gas of an interplanetary shock, Geophys. Res. Lett., 8, 173-176, 1981.

Berger, M. A. and Field, G. B.: The topological properties of magnetic helicity, J. Fluid Mech., 147, 147-148, 1984.

Bothmer, V. and Schwenn, R.: Eruptive prominences as sources of magnetic clouds in the solar wind, Space Science Reviews, 70, 215, 1994.

Bothmer V. and Rust M. D.: The field configuration of magnetic clouds and the solar cycle, in Coronal Mass Ejections, edited by Crooker, N., Joselyn, J. A, and Feynman, J., AGU, Washington D.C., Geophys. Monogr. 99, 137-146, 1997.

Bothmer, V. and Schwenn, R.: The structure and origin of magnetic clouds in the solar wind, Ann. Geophys., 16, 1-24, 1998, SRef-ID: 1432-0576/ag/1998-16-1.

Bothmer, V.: Sources of magnetic helicity over the solar cycle, in Proc. ISCS 2003 Symposium, "Solar variability as an input to the Earth's Environment”, Slovakia, ESA SP-535, 419-428, 2003.

Burlaga, L., Sittler, E., Mariani, F., and Schwenn, R.: Magnetic loop behind an interplanetary shock: Voyager, Helios and IMP 8 observations, J. Geophys. Res., 86, 6673-6684, 1981.

Burlaga, L. F. and Behannon, K. W.: Magnetic clouds: Voyager observations between 2 and 4 AU, Sol. Phys., 81, 181-192, 1982.

Burlaga, L.: Magnetic clouds and force-free fields with constant alpha, J. Geophys. Res., 93, 7217-7224, 1988.

Burlaga, L., Skoug, R. M., Smith, C. W., Webb, D. F., Zurbuchen, T. H., and Reinard, A.: Fast ejecta during the ascending phase of solar cycle 23: ACE observations, 1998-1999, J. Geophys. Res., 106, 20 957-20 977, 2001.

Burton, R. K., McPherron, R. L., and Russell, C. T.: An empirical relationship between interplanetary conditions and $D_{s t}$, J. Geophys. Res., 80, 4204-4214, 1975.

Cane, H. V. and Richardson, I. G.: Interplanetary coronal mass ejections in the near-Earth solar wind during 1996-2002, J. Geophys. Res., 108, doi10.1029/2002JA009817, SSH 6-1 - 6-13, 2003.

Cremades, H. and Bothmer, V.: On the three-dimensional configuration of coronal mass ejections, Astronomy and Astrophysics, 422, doi:10.1051/0004-6361:20035776, 307-322, 2004.

Elsässer, W. M.: Hydromagnetic dynamo theory, Rev. Mod. Phys., 28, 135-163, 1958.

Farrugia, C. J., Burlaga, L. F., Osherovich V. A., Richardson I. G., Freeman M. P., Lepping R. P., and Lazarus, A. J.: A study of an expanding interplanetary magnetic cloud and its interaction with the Earth's magnetosphere: The interplanetary aspect, J. Geophys. Res., 98, 7621-7632, 1993.

Fenrich, F. R. and Luhmann, J. G.: Geomagnetic response to magnetic clouds of different polarity, Geophys. Res. Lett., 25, 29993003, 1998.

Goldstein, H.: On the field configuration in magnetic clouds, in Solar Wind Five, edited by Neugebauer, M., Geophys. NASA Conf. Publ, 22, 731-733, 1983.

Gonzalez, W. T. and Tsurutani, B. T.: Criteria of interplanetary parameters causing intense magnetic storms $\left(D_{s t}<-100 \mathrm{nT}\right)$, Planet. Space Sci., 35, 1101-1109, 1987.

Gonzalez, W. D., Joselyn, J. A., Kamide, Y., Kroehl, H. W., Rostoker, G., Tsurutani, B. T., and Vasyliunas, V. M.: What is a geomagnetic storm, J. Geophys. Res., 99, 5771-5792, 1994.

Gopalswamy, N., Yashiro, S., Kaiser, M. L., Howard, R. A., and
Bougeret, J.-L.: Radio signatures of coronal mass ejection interaction: coronal mass ejection cannibalism?, Astronomy Journal, 548, 91-94, 2001.

Gosling, J. T. and McComas, D. J.: Field line draping about fast coronal mass ejecta: A source of strong out-of-ecliptic interplanetary magnetic fields, Geophys. Res. Lett., 14, 355-358, 1987.

Gosling, J. T.: Coronal mass ejections and magnetic flux ropes in interplanetary space, in Physics of magnetic flux ropes, Geophys. Monorg. Ser., edited by Russel, C. T., Priest, E. R., and Lee, L. C., AGU, Washington D.C, 58, 3518-3528, 1990.

Gosling, J. T., McComas, D. J., Phillips, J. L., and Bame, J.: Geomagnetic activity associated with Earth passage of interplanetary shock disturbances and coronal mass ejections, J. Geophys. Res., 96, 7831-7839, 1991.

Gosling, J. T., Birn, J., and Hesse, M.: Three-dimensional magnetic reconnection and the magnetic topology of coronal mass ejection events, J. Geophys. Res., 22, 869-872, 1995.

Hidalgo, M. A., Cid, C., Vinas, A. F., and Sequeiros, J.: A nonforce-free approach to the topology of magnetic clouds in the solar wind, J. Geophys. Res., 107, doi:10.1029/2001JA900100, SSH 1-1 - 1-7, 2002a.

Hidalgo, M. A., Nieves-Chinchilla, T., and Cid, C.: Elliptical crosssection model for the magnetic topology of magnetic clouds, Geophys. Res. Lett., 29, doi:10.1029/2001GL013875, SSH 15$1-15-4,2002 b$.

Hoeksema, J. D.: The heliospheric current sheet, The High Latitude Heliosphere, Proceedings of the 28th ESLAB Symposium, edited by Marsden, R. G., 1, 137-148, 1995.

Huttunen, K. E. J., Koskinen, H. E. J., and Schwenn, R.: Variability of magnetospheric storms driven by different solar wind perturbations, J. Geophys. Res., 107, doi:10.1029/2001JA900171, $1-12,2002 \mathrm{a}$.

Huttunen, K. E. J., Koskinen, H. E. J., Schwenn, R., and dal Lago, A.: Causes of major storms near the last solar maximum, in Proceeding of The 10th European Solar Physics Meeting "Solar variability: From core to outer frontiers", edited by Wilson, A., ESA Publication Division, ESTEC, 2, 137-140, 2002 b.

Huttunen, K. E. J. and Koskinen, H. E. J.: Importance of post-shock streams and sheath regions as driver of intense magnetospheric storms and high latitude activity, Ann. Geophys., 22, 1729-1738, 2004,

\section{SRef-ID: 1432-0576/ag/2004-22-1729.}

Hundhausen, A. J.: Sizes and Locations of Coronal Mass Ejections: SMM observations From 1980 and 1984-1989, J. Geophys. Res., 98, 13 177-13 200, 1993.

Kamide, Y., Yokoyama, N., Gonzalez, W., Tsurutani, B. T., Daglis, I. A., Brekke, A., and Masuda, S.: Two-step development of geomagnetic storms, J. Geophys. Res., 103, 6917-6921, 1998.

Klein, L. W. and Burlaga, L. F.: Interplanetary magnetic clouds at 1 AU, J. Geophys. Res., 87, 87 613-87 624, 1982.

dal Lago, A., Schwenn, R., and Gonzalez, W. D.: The halo CME expansion speed as a tool for predicting CME related shock travel time to 1 AU, Adv. Space R., 32, 2637-2640, 2003.

Lepping, R. P. and Behannon, K. W.: Magnetic field directional discontinuities, Minimum variance errors, J. Geophys. Res., 85, 4695-4703, 1980.

Lepping, R. P., Jones, J. A., and Burlaga, L. F.: Magnetic field structure of interplanetary magnetic clouds at $1 \mathrm{AU}, \mathrm{J}$. Geophys. Res., 95, 11 957-11965, 1990.

Lepping, R. P. and Berdichevsky, D.: Interplanetary magnetic clouds: Sources, properties, modelling, and geomagnetic relationship, Recent Res. Devel. Geophys., 3, 77-96, 2000. 
Lepping, R. P., Berdichevsky, D. B., Burlaga, L. F., Lazarus, A. J., Kasper, J., Desch, M. D., Wu, C. -C., Reames, D. V., Singer, H. J., Smith, C. W., and Ackerson, K. L.: The Bastille Day magnetic cloud and upstrem shocks: near-Earth interplanetary observations, Sol. Phys., 204, 287-305, 2001.

Liemohn, M. W., Kozyra, J. U., Thomsen, M. F., Roeder, J. L., Lu, G., Borovsky, J. E., and Cayton, T. E.: Dominant role of the asymmetric ring current in producing the stormtime $D_{s t}^{*}, \mathrm{~J}$. Geophys. Res., 106, 10 883-10 904, 2001.

Lundquist, S.: Magnetohydrostatic fields, Ark. Fys., 2, 316-365, 1950.

Lynch,B. J., Zurbuchen, T. H., Fisk, L. A., and Antiochos, S. K.: Internal structure of magnetic clouds: Plasma and composition, J. Geophys. Res., 108(A6), doi:10.1029/2002JA009591, SSH 6$1-6-14,2003$.

Martin, S. F. and McAllister, A. H.: Predicting the sign of magnetic helicity in erupting filaments and coronal mass ejections, Predicting the sign of magnetic helicity in erupting filaments and coronal mass ejections, in Coronal Mass Ejections, edited by N. Crooker and J. A Joselyn and Feynmann, AGU, Washington D.C., Geophys. Monogr. 99, 127-137, 1997.

Marubashi, K.: Interplanetary magnetic flux ropes and solar filaments, in Coronal Mass Ejections, edited by Crooker, N. and Joselyn, J. A. and Feynman, J., AGU, Washington D.C., Geophys. Monogr. 99, 147-156, 1997.

Mayaud, P. N.: Derivation Meaning, and Use of Geomagnetic Indices, AGU, Washington, D.C., Geophys. Monogr. 22, 115-128, 1980.

Mulligan, T., Russell, C. T. and Luhmann, J. G.: Solar cycle evolution of the structure of magnetic clouds in the inner heliosphere, Geophys. Res. Lett, 25, 2959-2962, 1998.

Mulligan, T. and Russell, C. T.: Multispacecraft modeling of the flux rope structure of interplanetary coronal mass ejections: cylindrically symmetric versus nonsymmetric solution, J. Geophys. Res., 106, 10 581-10 596, 2001.

O'Brien, T. P. and McPherron, R. L.: An empirical phase space analysis of ring current dynamics: Solar wind control of injection and decay, J. Geophys. Res., 105, 7707-7719, 2000a.

Osherovich, V. and Burlaga, L. F.: Magnetic clouds, in Coronal Mass Ejections, edited by N. Crooker and J. A Joselyn and Feynmann, AGU, Washington D.C., Geophys. Monogr. 99, 157-168, 1997.

Rust, D. M. and Kumar, A.: Helical magnetic fields in filaments, Sol. Phys., 155, 69-97, 1994.

Schwenn, R., dal Lago, A., Huttunen, E., and Gonzalez, W. D.: The association of coronal mass ejections with the effects of their counterparts near the Earth, in press, Ann. Geophys., 2005.
Sheeley, N. R., Howard, R. A., Koomen, M. J., Michelsand, D. J., Schwenn, R., Mahlhauser, K. H., and Rosenbauer, H.: Coronal mass ejections and interplanetary shocks, J. Geophys. Res., 90, 163-175, 1985.

Sonnerup, B. U. Ö. and Cahill, L. J.: Magnetopause structure and attitude from Explorer 12 observations, J. Geophys. Res., 72, 171-183, 1967.

Tandberg-Hanssen, E.: Solar prominences, 12, D. Reidel Publishing Company, Dordrecht-Holland/Boston, 1974.

Tripathi, D. K., Bothmer, V., and Cremades, H.: The basic characteristics of EUV post-eruptive arcades and their role as tracers of coronal mass ejection source regions, Astronomy and Astrophysic, 422, 337-349, 2003.

Tsurutani, B. T., Gonzalez, W. T., Tang, F., Akasofu, S. I., and Smith, E.: Origin of interplanetary southward magnetic fields responsible for major magnetic storms near solar maximum (19781979), J. Geophys. Res., 93, 8519-8531, 1988.

Tsurutani, B. T., Zhou, X.- Y., and Gonzalez, W. D.: A lack of substorm expansion phases during magnetic storms induced by magnetic cloud: The Storm-Substorm Relationship, Geophys. Monorg. Ser., edited by Sharma, S., Kamide, Y., and Lakhina, G. S., AGU, Washington, D.C., 124, 23-26, 2004.

Wang, C. B., Chao, J. K., and Lin, C.-H.: Influence of the solar wind dynamic pressure on the decay and injection of the ring current, J. Geophys. Res., 108, doi:10.1029/2003JA009851, SMP 5-1 - 5-10, 2003a.

Wang, Y. M., Ye, P. Z., and Wang, S.: Multiple magnetic clouds: several examples during March-April 2001, J. Geophys. Res., 108, doi:10.1029/2003JA009850, SSH 6-1 - 6-11, 2003b.

Wilson, R. M. and Hildner, E.: On the association of magnetic clouds with disappearing filaments, J. Geophys. Res., 91, 58675872, 1986.

Wu, C.-C., Lepping, R. P., and Gopalswamy, N.: Variations of magnetic clouds and CMEs with solar activity cycle, in Proc. ISCS 2003 Symposium, "Solar variability as an input to the Earth's Enviroment”, Slovakia, ESA, 1, 429-432, 2003.

Zhang, G. and Burlaga, L. F.: Magnetic clouds, geomagnetic disturbances, and cosmic ray decreases, J. Geophys. Res., 93, 25112518, 1988.

Zhao, X. P. and Hoeksema, J. T.: Central axial direction in magnetic clouds and its relation to southward interplanetary magnetic field events and depence on disappearing solar filaments, J. Geophys. Res., 103, 2077-2083, 1998. 